\title{
L-asparagine crystals with wide gap semiconductor features: Optical absorption measurements and density functional theory computations
}

G. Zanatta, C. Gottfried, A. M. Silva, E. W. S. Caetano, F. A. M. Sales, and V. N. Freire

Citation: The Journal of Chemical Physics 140, 124511 (2014); doi: 10.1063/1.4869179

View online: https://doi.org/10.1063/1.4869179

View Table of Contents: http://aip.scitation.org/toc/jcp/140/12

Published by the American Institute of Physics

\section{Articles you may be interested in}

Anhydrous crystals of DNA bases are wide gap semiconductors

The Journal of Chemical Physics 134, 175101 (2011); 10.1063/1.3584680

Hybrid functionals based on a screened Coulomb potential

The Journal of Chemical Physics 118, 8207 (2003); 10.1063/1.1564060

Molecular fractionation with conjugate caps for full quantum mechanical calculation of protein-molecule interaction energy

The Journal of Chemical Physics 119, 3599 (2003); 10.1063/1.1591727

Self-assembled peptide nanotubes as electronic materials: An evaluation from first-principles calculations Applied Physics Letters 106, 183707 (2015); 10.1063/1.4921012

Electronic transport in methylated fragments of DNA

Applied Physics Letters 107, 203701 (2015); 10.1063/1.4936099

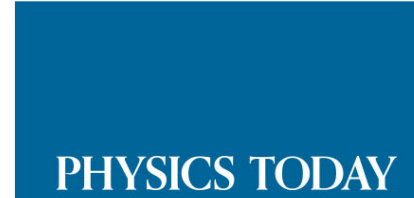

WHITEPAPERS
ADVANCED LIGHT CURE ADHESIVES

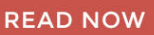

Take a closer look at what these environmentally friendly adhesive systems can do
PRESENTED BY

8)MASTERBOND' 


\title{
L-asparagine crystals with wide gap semiconductor features: Optical absorption measurements and density functional theory computations
}

\author{
G. Zanatta, ${ }^{1}$ C. Gottfried, ${ }^{1}$ A. M. Silva, ${ }^{2}$ E. W. S. Caetano, ${ }^{3, a)}$ F. A. M. Sales, ${ }^{4}$ \\ and V. N. Freire ${ }^{4}$ \\ ${ }^{1}$ Departamento de Bioquímica, Universidade Federal do Rio Grande do Sul, \\ 90035-003 Porto Alegre-RS, Brazil \\ ${ }^{2}$ Universidade Estadual do Piauí, 64260-000 Piripiri-Pi, Brazil \\ ${ }^{3}$ Instituto de Educação, Ciência e Tecnologia do Ceará, 60040-531 Fortaleza-CE, Brazil \\ ${ }^{4}$ Departamento de Física, Universidade Federal do Ceará, Caixa Postal 6030, 60455-760 Fortaleza-CE, Brazil
}

(Received 14 November 2013; accepted 27 February 2014; published online 31 March 2014)

\begin{abstract}
Results of optical absorption measurements are presented together with calculated structural, electronic, and optical properties for the anhydrous monoclinic L-asparagine crystal. Density functional theory (DFT) within the generalized gradient approximation (GGA) including dispersion effects (TS, Grimme) was employed to perform the calculations. The optical absorption measurements revealed that the anhydrous monoclinic L-asparagine crystal is a wide band gap material with $4.95 \mathrm{eV}$ main gap energy. DFT-GGA+TS simulations, on the other hand, produced structural parameters in very good agreement with X-ray data. The lattice parameter differences $\Delta a, \Delta b, \Delta c$ between theory and experiment were as small as $0.020,0.051$, and $0.022 \AA$, respectively. The calculated band gap energy is smaller than the experimental data by about $15 \%$, with a $4.23 \mathrm{eV}$ indirect band gap corresponding to $\mathrm{Z} \rightarrow \Gamma$ and $\mathrm{Z} \rightarrow \beta$ transitions. Three other indirect band gaps of $4.30 \mathrm{eV}, 4.32 \mathrm{eV}$, and $4.36 \mathrm{eV}$ are assigned to $\alpha 3 \rightarrow \Gamma, \alpha 1 \rightarrow \Gamma$, and $\alpha 2 \rightarrow \Gamma$ transitions, respectively. $\Delta$-sol computations, on the other hand, predict a main band gap of $5.00 \mathrm{eV}$, just $50 \mathrm{meV}$ above the experimental value. Electronic wavefunctions mainly originating from $\mathrm{O} 2 p$-carboxyl, C $2 p$-side chain, and C $2 p$-carboxyl orbitals contribute most significantly to the highest valence and lowest conduction energy bands, respectively. By varying the lattice parameters from their converged equilibrium values, we show that the unit cell is less stiff along the $b$ direction than for the $a$ and $c$ directions. Effective mass calculations suggest that hole transport behavior is more anisotropic than electron transport, but the mass values allow for some charge mobility except along a direction perpendicular to the molecular layers of L-asparagine which form the crystal, so anhydrous monoclinic L-asparagine crystals could behave as wide gap semiconductors. Finally, the calculations point to a high degree of optical anisotropy for the absorption and complex dielectric function, with more structured curves for incident light polarized along the 100 and 101 directions. (C 2014 AIP Publishing LLC. [http://dx.doi.org/10.1063/1.4869179]
\end{abstract}

\section{INTRODUCTION}

Asparagine (Asn, N), with chemical formula $\mathrm{C}_{4} \mathrm{H}_{8} \mathrm{~N}_{2} \mathrm{O}_{3}$, is one of the 20 amino acids which form proteins in living beings. It has an essential role on the biosynthesis of glycoproteins and, under physiological conditions, destabilizes and spontaneously deamidates into aspartic acid (Asp), which limits the lifetime of proteins through a degradation process that affects biological functions, ${ }^{1,2}$ leading to diseases (for example, deamidation accelerates amyloid formation ${ }^{3}$ ), and deteriorating therapeutic proteins. ${ }^{4}$ The reaction pathways of Asn deamidation were investigated within the density functional theory (DFT) framework, being unveiled their $\mathrm{pH}$-dependent mechanism, ${ }^{5}$ the effect of solvent molecules, ${ }^{6}$ and their role in nonenzymatic peptide bond cleavage at asparagine sites. $^{7}$ Besides, Asn deamidation is a key aspect related to the stability of protein pharmaceuticals ${ }^{4}$ and proteomic sample preparation. ${ }^{8}$ The Asn deamidation reaction path and its multitude of subproducts is related to the ring

\footnotetext{
${ }^{a)}$ Electronic mail: ewcaetano@gmail.com
}

formation tendency of the asparagine molecular structure (see top right side and middle of Fig. 1). Thus, it would be very helpful to achieve a deeper understanding of the structural features of asparagine in vacuum, aqueous media, and in the solid state.

A systematic and extensive conformational search has been performed by Chen et al. ${ }^{9}$ for gas phase asparagine using the B3LYP/6-311G* functional/basis set combination. By allowing for all combinations of internal single-bond rotamers, they have obtained as much as 972 unique trial structures; after further optimization, a total of 62 conformers were found, with the global energy minimum being stabilized by four hydrogen bonds $\mathrm{COOH} \cdots \mathrm{NH}_{2}, \mathrm{NH}_{2} \cdots \mathrm{CO}\left(\mathrm{NH}_{2}\right)$, $\mathrm{C}=\mathrm{O} \cdots \mathrm{NH}_{2}$, and $(\mathrm{CO}) \mathrm{NH}_{2} \cdots \mathrm{CO}(\mathrm{OH})$, forming a network of intramolecular interactions, as shown at the top and middle of Fig. 1. Recently, a theoretical/experimental matrixisolation FT-IR study of the conformational behavior and $\mathrm{H}-$ bond structure of asparagine was undertaken by Boeckx and Maes,${ }^{10}$ which found ten stable low energy asparagine conformations (total energy variation smaller than $10 \mathrm{~kJ} \mathrm{~mol}^{-1}$ ), the most stable containing only 3 intramolecular H-bonds: 

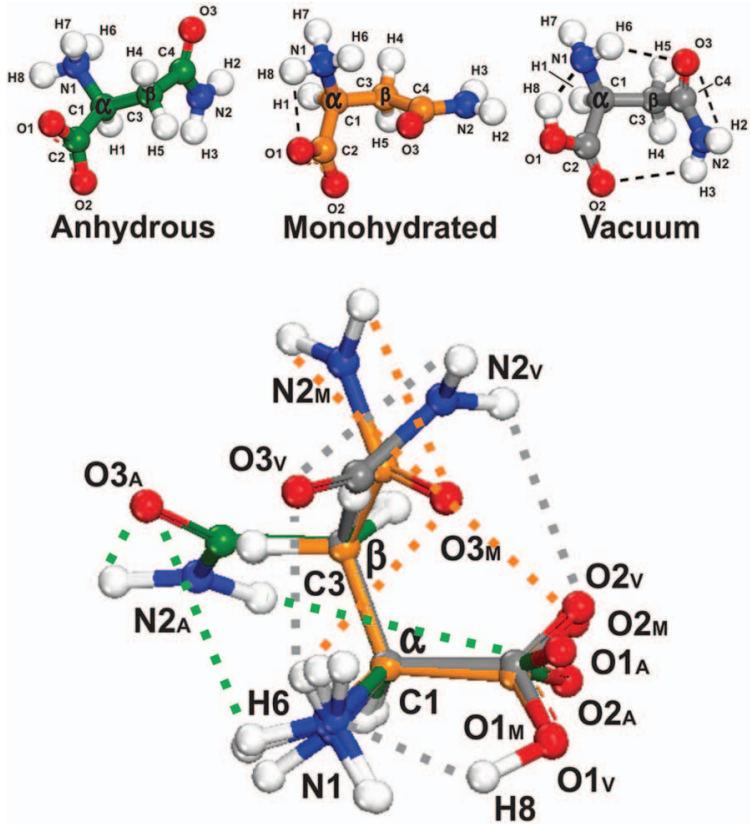

\begin{tabular}{lcccc}
\hline & Cabezas & Vacuum & Anhydrous & Monohydrated \\
\hline \hline O1H8...N1 & 1.86 & 1.90 & - & - \\
N1H6...O3 & 2.18 & 2.22 & 3.18 & 2.56 \\
N2H2...O3 & 2.50 & 2.50 & 2.55 & 2.52 \\
N2H3...O2 & 2.47 & 2.71 & 4.07 & 4.80 \\
\hline
\end{tabular}

FIG. 1. Top: Asparagine molecules in anhydrous monoclinic (A, green backbone) and monohydrated orthorhombic ( $\mathrm{M}$, orange backbone) asparagine crystals, according to the X-ray data of Yamada et al. ${ }^{13}$ and Verbist et al. ${ }^{16}$ respectively. The DFT-calculated Asn molecular structure in vacuum (V, gray backbone) by Chen et al. ${ }^{9}$ is also shown; middle: superposition of the molecular configurations depicted at the top obtained through the alignment of the $C_{\alpha}-C_{\beta}$ bond; bottom: intramolecular distances (in $\AA$ ) for the atoms involved in the formation of hydrogen bonds present in the anhydrous and monohydrated Asn crystals, as well as in the vacuum lowest energy conformer. The hydrogen bond lengths obtained by Cabezas et al. ${ }^{11}$ are also shown for the sake of comparison.

$\mathrm{C}=\mathrm{O}^{s c} \cdots \mathrm{HN}^{b b}, \mathrm{C}=\mathrm{O}^{b b} \cdots \mathrm{HN}^{s c}$, and $\mathrm{OH}^{b b} \cdots \mathrm{N}^{b b}$. On the other hand, Cabezas et al. ${ }^{11}$ by employing a combination of laser ablation (LA) with molecular beam (MB) and Fourier transform microwave (FTMW) spectroscopy, together with ab initio theoretical computations to predict spectroscopic parameters for direct comparison with the experimental ones, investigated the conformation locking of asparagine, concluding that the lowest energy Asn conformer exists in the gas phase as a result of a network of four cooperative contacts $\mathrm{N}_{\alpha} \cdots \mathrm{HO}, \mathrm{O}_{\beta} \cdots \mathrm{H}-\mathrm{N}_{\alpha}, \mathrm{N}_{\beta} \mathrm{H} \cdots \mathrm{O}_{\beta}$, and $\mathrm{O}_{\alpha} \cdots \mathrm{HN}_{\beta}$ with hydrogen bond lengths of $1.86 \AA, 2.18 \AA$, $2.50 \AA$, and $2.47 \AA$, respectively. Such hydrogen bond network was also observed by Chen et al., ${ }^{9}$ as depicted in the top part of Fig. 1, vacuum geometry.

With the purpose of investigating the causes behind such conformational preference of asparagine in the gas phase and the interaction between asparagine and solvent molecules in terms of cage effects, Selvaraj et al. ${ }^{12}$ carried out classical and Car-Parrinello molecular dynamics simulations to study the solvent effect on the Asn conformational space. A solvent polarization-induced conformational unlocking of asparagine in aqueous solutions was discerned, as the solvent polarity induced a break up of $\mathrm{NH} \cdots \mathrm{O}=\mathrm{C}$ bonding between the side chain and the terminal groups of asparagine, but without affecting the hydrogen bonds within the side chain and the terminal groups.

In the solid state, intermolecular hydrogen bonds unlock asparagine as well, according to X-ray diffraction data of both anhydrous monoclinic $P 2_{1}(\mathrm{Z}=2)^{13}$ and monohydrated orthorhombic $P 2{ }_{1} 2_{1} 2_{1}(Z=4)^{14-16}$ crystalline structures (see again the top and middle parts of Fig. 1). The $\mathrm{N}_{2} \mathrm{H}_{3} \cdots \mathrm{O}_{2}$ and $\mathrm{N}_{1} \mathrm{H}_{6} \cdots \mathrm{O}_{3}$ hydrogen bond lengths increase significantly, which implies in an unlocking effect, as one can see at the bottom table of Fig. 1. Actually, the $\mathrm{N}_{2} \mathrm{H}_{3} \cdots \mathrm{O}_{2}\left(\mathrm{~N}_{1} \mathrm{H}_{6} \cdots \mathrm{O}_{3}\right)$ hydrogen bond is strongly (moderately) weakened, while the $\mathrm{N}_{2} \mathrm{H}_{2} \cdots \mathrm{O}_{3}$ is kept by the lattice intermolecular interaction. The molecular unlocking leads to Asn molecular structures in crystals that are remarkably different from those observed in vacuum and water solutions. Consequently, one cannot use the smallest energy isolated conformer (even in solution) to calculate the crystalline properties accurately.

For this reason, efforts to describe theoretically the role of intermolecular bonds on the properties of molecular crystals were made in the last few years. ${ }^{17-24}$ As members of the class of molecular crystals, anhydrous monoclinic and monohydrated orthorhombic asparagine crystals are stabilized by hydrogen bonds and van der Waals interactions which determine their structural, electronic, optical, and vibrational properties. Research on molecular crystals has being somewhat restricted to experimental characterization of their polymorphs through infrared, Raman, nuclear magnetic resonance (NMR), and electron paramagnetic resonance (EPR) spectroscopies, which are very useful in the pharmaceutical domain, for example. On the theoretical side, the computational cost required to study such systems within the DFT formalism has restrained advances in the field, as stated by Tulip and Clark. ${ }^{17}$ Even the numerous spectroscopic measurements on molecular crystals are usually explained by appealing to the properties of the individual molecules, i.e., lattice effects being disregarded. However, the increase of computational power and the development of new DFT codes have allowed the application of these simulation methods to systems with up to hundreds of atoms. ${ }^{17}$

As our research group has pointed out in previous works, ${ }^{18,19}$ some attempts to employ amino acid films in biosensors and optoelectronic devices are being made. For example, a study of the adhesion of amino acids on a series of inorganic surfaces including insulators and semiconductors was performed by Willett et al. ; $^{25}$ the adsorption footprints of proline on $\mathrm{Cu}(110)$ was studied, ${ }^{26}$ and a reactive dynamics simulation of monolayer and multilayer adsorption of glycine on $\mathrm{Cu}(110)$ was carried out; ${ }^{27}$ the adsorption and ordering of L-tyrosine and L-cysteine on the $\operatorname{Ag}(111)$ surface was studied through several techniques by Reichert et al. ${ }^{28}$ and Fischer et al. ${ }^{29}$ respectively, and the adsorption of glutamic acid on $\operatorname{Ag}(100)$ was surveyed by Smerieri et al.,$^{30}$ self-assemblies of $\mathrm{D}$ - and L-methionine on $\mathrm{Au}(111)$ surfaces were studied by Naitabdi and Humblot, ${ }^{31}$ and the hydrogen-bonded network of histidine on $\mathrm{Ge}(100)$ was examined as well. ${ }^{32} \mathrm{On}$ the other hand, Oda and Nakayama ${ }^{33,34}$ have proposed to 
control electrically the amino acid ionization and the conformation of proteins arranged on semiconductor surfaces, which might produce new types of biodevices. Besides, Stroscio and Dutta ${ }^{35}$ described advances in man-made nanostructures integrated with biological molecules and systems, their properties, characteristics, and functions. Thus, the understanding of fundamental aspects (electronic and optical characteristics, particularly) of amino acid crystals and films is of paramount importance for the future development of biooptoelectronic devices.

Among the few reports already published concerning the field of amino acid crystal simulations, some of them have presented results suggesting that alanine, leucine, isoleucine, and glycine ${ }^{17-19}$ are wide band gap semiconductors, while valine and cysteine could be small band gap isolators, ${ }^{17,20}$ on the other hand, it was suggested that anhydrous L-aspartic crystals behave as wide-gap semiconductors with higher carrier mobilities for directions parallel to the L-aspartic acid molecular layers, ${ }^{21}$ while anhydrous L-serine crystals behave like n-type wide gap semiconductors. ${ }^{22}$ Besides, it was also pointed out that anhydrous crystals of DNA bases are wide gap semiconductors. ${ }^{23}$ The characterization of the band gap of these molecular materials and their charge transport properties, which depends on their band structures, are relevant for the development of bioorganic electronic and optoelectronic devices, ${ }^{36}$ as well as bionanoelectronics, ${ }^{37}$ which could advance the development of sustainable, biodegradable, biocompatible, low-cost, and mass production electronic components.

The purpose of this work is to present the results of DFT calculations of the structural, electronic, and optical properties of anhydrous monoclinic L-asparagine crystals. Optical absorption measurements in these crystals were also performed, from which a wide indirect band gap of $4.95 \mathrm{eV}$ was obtained, which is close to the DFT-estimated $4.23 \mathrm{eV}$ value assigned to $\mathrm{Z} \rightarrow \Gamma$ and $\mathrm{Z} \rightarrow \beta$ transitions between the uppermost valence band and the lowermost conduction band. Carrier effective masses estimated from parabolic fittings of the band curves indicate that holes in anhydrous monoclinic L-asparagine crystals can behave as in a semiconductor, while the electrons behave as in an insulator. Finally, the calculated optical absorption and dielectric function along some symmetry directions display a noticeable anisotropy for polarized incident light.

\section{OPTICAL ABSORPTION MEASUREMENT}

Anhydrous monoclinic L-asparagine powder with purity $>99 \%$ was purchased from Sigma-Aldrich. X-ray diffraction measurements were accomplished to confirm the monoclinic crystal structure (data not presented here). The powder was mixed with $\mathrm{KBr}$ to form anhydrous L-Asn- $\mathrm{KBr}$ pellets. Light absorption measurements were carried out in these pellets using a Varian Cary 5000 UV-visible NIR spectrophotometer equipped with solid sample holders. The absorption spectra of the samples were recorded in the $200-800 \mathrm{~nm}$ wavelength range (6.21-1.55 eV), with background removal and baseline corrections made when necessary.

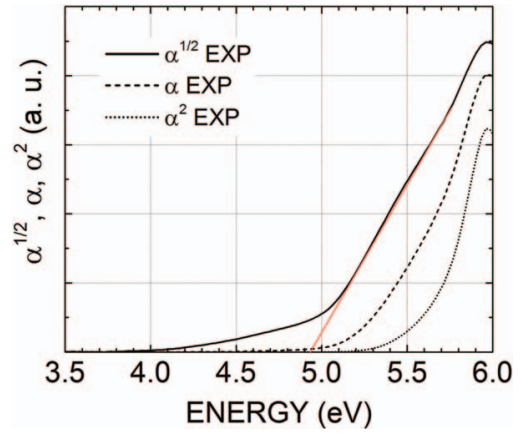

FIG. 2. Optical absorption $(\alpha)$, optical absorption squared $\left(\alpha^{2}\right)$, and square root $\left(\alpha^{1 / 2}\right)$ of L-Asn-KBr pellets measured at $300 \mathrm{~K}$ with nonpolarized incident radiation. The red straight line is used to estimate the indirect band gap.

The onset of the optical absorption $\alpha(\varepsilon)$ as a function of the incident photon energy $\varepsilon$ in an indirect gap crystal varies with $\varepsilon^{2}$, while for a direct gap material it increases with $\varepsilon^{1 / 2} \cdot 38,39$ Hence, if the square root (square) of the optical absorption displays a linear behavior as a function of the energy, the material must have an indirect (direct) electronic main band gap. With these characteristics in mind and looking now to Fig. 2, one can see plots for $\alpha^{1 / 2}, \alpha$, and $\alpha^{2}$ for the anhydrous L-Asn- $\mathrm{KBr}$ pellets. One can observe that $\alpha^{2}$ has a curved onset shape beginning at about $5.2 \mathrm{eV}$, while the $\alpha$ curve onset starts at about $4.5 \mathrm{eV}$. The curve for $\alpha^{1 / 2}$, in contrast, exhibits an onset at about $4.0 \mathrm{eV}$ with a smooth linear increase up to $5.0 \mathrm{eV}$ (which we believe is an artifact of the square rooting process, as the optical absorption in this energy range is very small) and a sharp linear increase between 5.0 $\mathrm{eV}$ and $5.7 \mathrm{eV}$. Taking into account these curves, we estimate that the L-Asn crystal has an indirect band gap of $4.95 \mathrm{eV}$, which corresponds to the energy at the point where the red straight line tangent to the $\alpha^{1 / 2}$ curve intercepts the energy axis.

As we will see later, a main indirect band gap is also predicted from our DFT computations. Indeed, previous optical absorption measurements in amino acid crystals indicate that these systems tend to exhibit indirect band gaps. ${ }^{19-22} \mathrm{~A}$ comparison of the energy gap of anhydrous monoclinic Lasparagine crystal with the values obtained for other amino acid crystals shows that it is smaller than the estimated for monoclinic $\left(P 2_{1} / n, \mathrm{Z}=4\right) \alpha$-glycine crystals, $5.11 \mathrm{eV},{ }^{19}$ and larger than the values for orthorhombic $\left(P 2_{1} 2_{1} 2_{1}, Z=4\right) \mathrm{L}$ cysteine crystals, $4.62 \mathrm{eV} ;{ }^{20}$ it is also close to the $5.02 \mathrm{eV}$ gap measured for anhydrous monoclinic $\left(P 2_{1}, \mathrm{Z}=2\right)^{21} \mathrm{~L}$ aspartic acid crystals, and much smaller than the $5.90 \mathrm{eV}$ measured gap for anhydrous monoclinic $\left(P 22_{1} 2_{1} 2_{1}, \mathrm{Z}=4\right) \mathrm{L}$ serine crystals. ${ }^{22}$ The L-asparagine monoclinic crystal has a wide gap, anyway, but the values of the effective masses of its charge carriers are also necessary to know its charge transport properties (see Sec. V).

\section{DFT COMPUTATIONAL DETAILS}

The density functional computations were carried out using the CASTEP code. ${ }^{40}$ Lattice parameters and atomic 
positions of anhydrous monoclinic L-asparagine crystals as measured by Yamada et al. ${ }^{13}$ were used to produce the input unit cell structure, which belongs to the $P 2_{1}$ space group and has $\mathrm{Z}=2$. Experimental lattice parameters are $a=5.0622(4)$ $\AA, b=6.7001(5) \AA, c=8.0543(5) \AA, V=273.06(3) \AA^{3}$, $\beta=91.706(5)^{\circ}$. It was necessary to perform a DFT geometry optimization to evaluate both the unit cell parameters and atomic coordinates for the sake of consistency, and to improve the accuracy of experimental data with respect to the hydrogen atom positioning. For example, in the case of the vaterite $\mathrm{CaCO}_{3}$ crystal significant differences were observed in the spatial pattern of the carbonate groups inside of the unit cell between experiment and DFT calculations. ${ }^{41,42}$ Besides, an adequate geometry optimization is essential to calculate the vibrational properties, from which one can derive the infrared and Raman spectra which are dependent on the crystal geometry, amino acid side chain type, hydrogen bonding pattern, and van der Waals interactions. Optoelectronic properties are also sensitive to geometry optimization, as the electronic band structure varies appreciably when the lattice parameters and the internal atomic coordinates are changed.

The carbon backbone of the L-asparagine molecules in the crystalline phase is practically planar, with average deviation from planarity being smaller than $0.01 \AA$. Experimental values for the unit cell parameters from Yamada et al. ${ }^{13}$ are given in Table I. The L-asparagine zwitterionic molecule is depicted in Fig. 3(a), while the monoclinic unit cell of anhydrous monoclinic L-asparagine crystals is shown in Fig. 3(b). Parallel layers of $\mathrm{L}$-asparagine molecules form planes along the 101 direction as depicted in Fig. 3(c); adjacent layers interact through hydrogen bonds occurring between charged groups $\left(\mathrm{CO}^{-}, \mathrm{NH}_{3}{ }^{+}\right.$, and $\left.\mathrm{COO}^{-}\right)$. Within a single layer, the molecules are linked forming zigzag chains created by hydrogen bonds involving the $\mathrm{NH}_{3}{ }^{+}$group. Finally, intermolecular "tunnels" are present in the L-asparagine lattice structure (see Fig. 3(d)).

The DFT calculations ${ }^{43,44}$ were carried using two distinct approaches for the exchange-correlation functional: the local density approximation (LDA) using the Cerpeley-Alder-

TABLE I. Lattice parameters (LP) in $(\AA)$, unit cell volume in $\left(\AA^{3}\right)$, and unit cell angle $\beta$ (deg) for the anhydrous monoclinic L-asparagine crystal. The LDA and GGA+TS calculation results were obtained using 500, 830, and $1100 \mathrm{eV}$ cutoff energies. Their deviations $\Delta$ in comparison with experimental data ${ }^{13}$ are also shown.

\begin{tabular}{lrrrccc}
\hline \hline $\mathrm{LP}$ & $\mathrm{L}_{500}$ & $\mathrm{~L}_{830}$ & $\mathrm{GTS}_{500}$ & $\mathrm{GTS}_{830}$ & $\mathrm{GTS}_{1100}$ & Exp. \\
\hline $\mathrm{a}(\AA)$ & 5.222 & 4.941 & 5.454 & 5.082 & 5.081 & 5.062 \\
$\Delta \mathrm{a}(\AA)$ & 0.160 & -0.121 & 0.392 & 0.020 & 0.019 & $\ldots$ \\
$\mathrm{b}(\AA)$ & 6.929 & 6.507 & 7.144 & 6.751 & 6.750 & 6.700 \\
$\Delta \mathrm{b}(\AA)$ & 0.229 & -0.193 & 0.444 & 0.051 & 0.050 & $\ldots$ \\
$\mathrm{c}(\AA)$ & 7.984 & 7.883 & 8.177 & 8.076 & 8.074 & 8.054 \\
$\Delta \mathrm{c}(\AA)$ & -0.070 & -0.172 & 0.123 & 0.022 & 0.020 & $\ldots$ \\
$\mathrm{V}\left(\AA^{3}\right)$ & 288.7 & 253.3 & 318.3 & 277.0 & 276.8 & 273.1 \\
$\Delta \mathrm{V}\left(\AA^{3}\right)$ & 15.68 & -19.78 & 45.22 & 3.980 & 3.770 & $\ldots$ \\
$\beta(\mathrm{deg})$ & 88.20 & 92.06 & 87.30 & 91.30 & 91.3 & 91.71 \\
$\Delta \beta(\mathrm{deg})$ & -3.502 & 0.349 & -4.402 & -0.407 & -0.420 & $\ldots$ \\
\hline \hline
\end{tabular}
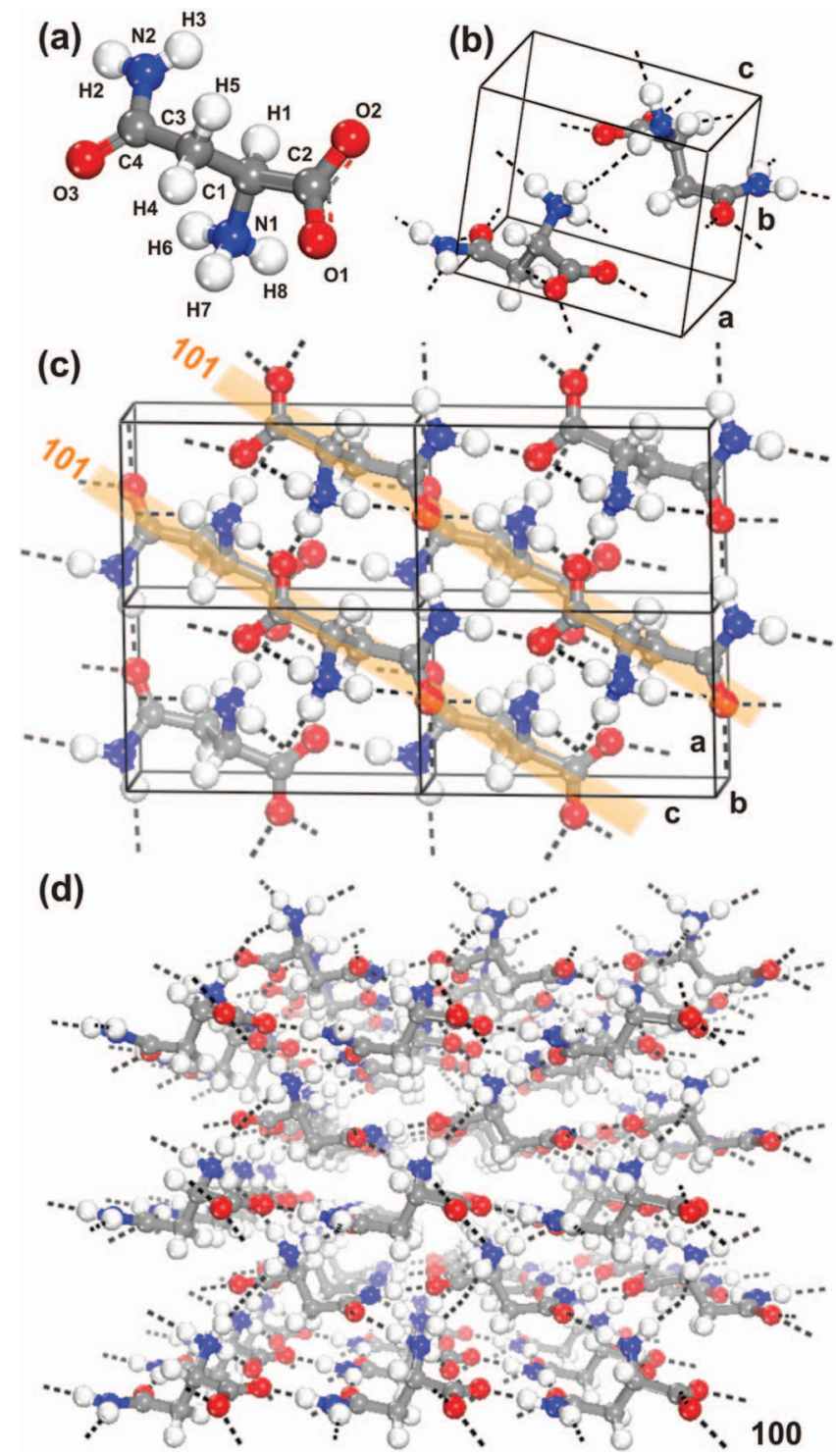

FIG. 3. (a) L-asparagine zwitterionic molecule; (b) anhydrous L-asparagine monoclinic unit cell; (c) view of two superposed 101 (ac) planes of Lasparagine zwitterionic molecules; (d) intermolecular tunnels in anhydrous L-asparagine monoclinic crystals.

Perdew-Zunger ${ }^{45,46}$ parameters (CAPZ), and the generalized gradient approximation (GGA) employed the Perdew-BurkeErnzerhof (PBE) ${ }^{47}$ functional. We have also performed GGAPBE simulations modified with the extra dispersion correction scheme (GGA+TS) proposed by Tkatchenko and Scheffler ${ }^{48}$ and, for the sake of comparison, the GGA-PBE functional including the dispersion semiempirical construct of Grimme. ${ }^{49}$ While the PBE functional leads to results close to the ones obtained by using the PW91 functional, ${ }^{50}$ dispersion corrections avoid the need to use high-level quantum methods to describe van der Waals interactions. Norm-conserving pseudopotentials ${ }^{51}$ were used to replace the core electrons in each atomic species, with valence electronic configurations $\mathrm{C}-2 s^{2} 2 p^{2}, \mathrm{~N}-2 s^{2} 2 p^{3}$, and $\mathrm{O}-2 s^{2} 2 p^{4}$. Each unit cell has 140 electrons (36 core and 104 valence). A Monkhorst-Pack ${ }^{52} 4$ $\times 4 \times 4$ sampling was employed to evaluate integrals in the reciprocal space, which was more than enough to give a well 
converged electronic structure. For the optical properties calculations, this sampling was increased to $10 \times 10 \times 10$, as it is necessary to use more k-points in the Brillouin zone (BZ) when running optical matrix element calculations (the matrix elements are more sensitive to the Brillouin zone sampling than electronic energies).

Lattice parameters, angles, and atomic positions were optimized by searching for a total energy minimum. In order to perform the geometry optimization, the following convergence thresholds were considered along successive self-consistent field computation steps: total energy change smaller than $0.5 \times 10^{-5} \mathrm{eV} /$ atom, maximum force per atom below $0.01 \mathrm{eV} / \AA$, pressure smaller than $0.02 \mathrm{GPa}$, and maximum atomic displacement below $0.5 \times 10^{-3} \AA$. The BFGS minimizer ${ }^{53}$ was employed to carry out the unit cell optimization. In the BFGS scheme, a starting Hessian is recursively updated. Self-consistent field convergence thresholds were: total energy/atom varying by less than $0.5 \times 10^{-6} \mathrm{eV}$, electronic eigenenergy varying by less than $0.1250 \times 10^{-6} \mathrm{eV}$ at most, with a convergence window of 3 cycles. A plane-wave basis set represents the Kohn-Sham orbitals, with cutoff energies of 500 and $830 \mathrm{eV}(500,830$, and $1000 \mathrm{eV})$ for the LDA (GGA+TS) exchange-correlation functional, with the final value selected after performing a convergence study to ensure the absence of imaginary vibrational frequencies. The quality of this basis set is kept fixed as the unit cell volume evolves during the geometry optimization process.

The Kohn-Sham electronic band structure and the electronic density of states (total and partial per orbital and per atom) were evaluated for both the optimized LDA and GGA+TS crystals (the LDA results are omitted since the GGA+TS ones are considerably better), as well as the dielectric function and optical absorption for polarized light along a set of chosen crystalline planes and in the case of a polycrystalline sample. Effective masses at the extrema of the valence and conduction bands were estimated by quadratic interpolation of the corresponding band curves. It is well known that Kohn-Sham calculations tend to severely underestimate solid state band gaps. ${ }^{54}$ In order to amend for that, we have employed the $\Delta$-sol method proposed by Chan and Ceder, ${ }^{55}$ which extended the Delta self-consistent-field $(\triangle \mathrm{SCF})$ approach for molecules to crystals taking into account total energy differences and electronic dielectric screening to obtain much better gap estimates (their proposal achieves a 70\% decrease of mean absolute errors in comparison with KohnSham gaps for over 100 compounds) with cheap computational cost. The complex dielectric function $\epsilon(\omega)=\operatorname{Re}(\epsilon)+i$ $\operatorname{Im}(\epsilon)$ and the optical absorption $\alpha(\omega)$ of anhydrous monoclinic L-asparagine crystals were calculated following the same scheme described in a previous work. ${ }^{19}$

\section{STRUCTURAL PROPERTIES}

A convergence study of the monoclinic L-asparagine unit cell structure was performed by increasing the planewave cutoff energy from $500 \mathrm{eV}$ to $830 \mathrm{eV}$, and then to $1100 \mathrm{eV}$. The optimized unit cell parameters obtained using the GGA+TS exchange-correlation energy are shown in the columns $\mathrm{GTS}_{500}, \mathrm{GTS}_{830}$, and $\mathrm{GTS}_{1100}$ of Table I, respec- tively. For the sake of comparison, the results obtained using the LDA exchange-correlation functional for cutoff energies of $500 \mathrm{eV}$ and $830 \mathrm{eV}$ are shown in columns $\mathrm{L}_{500}$ and $\mathrm{L}_{830}$, respectively, together with the experimental values from Yamada et al. ${ }^{13}$ One can observe that the DFT-LDA calculated unit cell parameters at $830 \mathrm{eV}(a=4.941 \AA, b=6.5072 \AA$, and $c=7.883 \AA$ ) are smaller than the X-ray values (see the deviations $\Delta a, \Delta b, \Delta c$ relative to the measurements), which is due to the LDA functional trend to overestimate interatomic forces. Nevertheless, this is also the reason by which the LDA approximation can be useful to study systems where weak intermolecular interactions are relevant. ${ }^{23,24,56,57}$

In contrast, pure GGA functionals are able to provide a good description of hydrogen bonds, but tend to underestimate the strength of interatomic interactions, and some test simulations we accomplished (not shown here) suggested a lack of accuracy in predicting the structural parameters of L-asparagine crystals. After adding the extra dispersion correction scheme proposed by Tkatchenko and Scheffler ${ }^{48}$ $(\mathrm{GGA}+\mathrm{TS})$, however, we have improved the estimated values for the unit cell parameters, compensating for the GGA underbinding effect. From Table I, one can see that the lattice parameters and unit cell volume are remarkably nice for $830 \mathrm{eV}$, practically remaining the same for the $1100 \mathrm{eV}$ cutoff energy. The deviations relative to the X-ray measurements are nearly $0.02 \AA(0.05)$ for $\Delta a$ and $\Delta c(\Delta b)$, and smaller than $4.0 \AA^{3}$ for the unit cell volume variation $\Delta V$. Using the Grimme dispersion corrected GGA-PBE functional, ${ }^{49}$ we also obtained good estimates for the lattice parameters, with $a=5.05 \AA, b=6.66 \AA$, and $c=8.03 \AA$, with deviations relative to experiment of $0.01 \AA(\Delta a), 0.04 \AA(\Delta b)$, and $0.02 \AA(\Delta c)$. The GGA+TS (GGA+Grimme) estimate for the $\beta$ angle was $91.3^{\circ}\left(92.3^{\circ}\right)$, very close to the experimental value $\left(91.71^{\circ}\right)$.

All vibrational normal modes obtained for the crystal geometry with a cutoff energy of $830 \mathrm{eV}$ exhibited positive frequencies, indicating that geometry optimization to a total energy minimum was reached (in a work to be published soon we intend to present the results of the vibrational analysis for the infrared and Raman spectra of asparagine anhydrous crystals). Thus, we have used this cutoff energy to calculate the electronic and optical properties of the anhydrous monoclinic asparagine crystal to avoid extra computational costs. Fractional atomic coordinates, selected bond lengths, bond angles, and torsion angles, as well as hydrogen bond related lengths and angles for the calculated (GGA+TS and LDA) and the experimental data are included in the supplementary material (Tables I-IV) ${ }^{58}$ showing that the GGA+TS results are in better agreement with experiment than those of LDA.

In order to approximately evaluate the stiffness of the anhydrous monoclinic asparagine crystal, the lattice parameters were slightly varied about their converged values. Total energy values $E\left(\Delta a / a_{0}\right), E\left(\Delta b / b_{0}\right)$, and $E\left(\Delta c / c_{0}\right)$ are depicted in Fig. 4, being obtained by performing single point energy calculations after rescaling one lattice parameter and keeping the other two constant as well as the atomic fractional coordinates. One can note that the total energy change for $E\left(\Delta b / b_{0}\right)$ is much smaller than those of $E\left(\Delta a / a_{0}\right)$ and $E\left(\Delta c / c_{0}\right)$, which is probably due to the dominance of intramolecular 


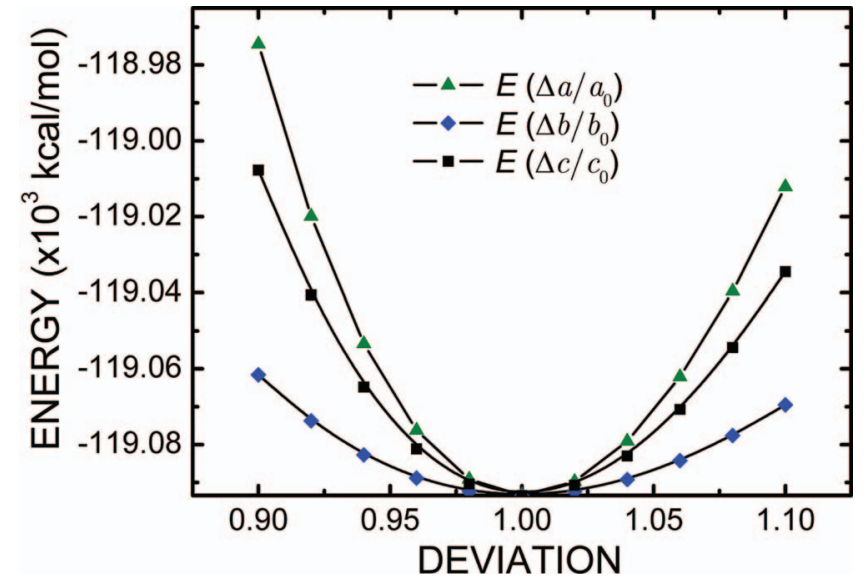

FIG. 4. GGA+TS total energy $E$ of the anhydrous monoclinic L-asparagine crystal unit cell as a function of the lattice parameter relative deviations $\Delta a / a_{0}, \Delta b / b_{0}, \Delta c / c_{0}$ relative to the optimal parameters $a_{0}, b_{0}$, and $c_{0}$, respectively, for a plane-wave cutoff energy of $1100 \mathrm{eV}$.

covalent bonds disposed along the $a$ and $c$ directions (see Fig. 3(c)), while along $b$ hydrogen bonds and just a few covalent bonds (mainly $\mathrm{C} 1-\mathrm{C} 3$ ) seem to be deformed in a remarkable way. The arrangement of hydrogen bonds suggest that, if the monoclinic asparagine crystal were dismantled by removing one molecular layer each time, it would be easier to remove molecular planes along the $b$ direction in comparison with planes along $a$ and $c$.

\section{ELECTRONIC PROPERTIES}

The Kohn-Sham band structure describes the electron eigenenergies $E_{n}(\mathbf{k})$ as a function of a set of quantum numbers that form the components of a wave vector $\mathbf{k}=\left(\mathbf{k}_{1}, \mathbf{k}_{2}\right.$, $\mathbf{k}_{3}$ ) ( $n$ is the band number). Usually the first BZ is sampled by calculating these eigenenergies along a path containing a selected set of high-symmetry points. For the anhydrous monoclinic L-asparagine crystal, the path in the BZ used for the band structure computations is shown in Fig. 5. The following set of high-symmetry points of interest was chosen: $\Gamma(0,0,0), \mathrm{Z}(0,0,1 / 2), \mathrm{Y}(0,1 / 2,0), \mathrm{A}(-1 / 2,1 / 2,0), \mathrm{B}(-1 / 2,0,0)$, $\mathrm{C}(0,1 / 2,1 / 2), \mathrm{D}(-1 / 2,0,1 / 2)$, and $\mathrm{E}(-1 / 2,1 / 2,1 / 2)$. Other non-symmetry points as $\alpha_{1}=(-0.24,0.00,0.24), \alpha_{2}$ $=(-0.22,0.22,0.22), \quad \alpha_{3}=(0.00,0.42,0.42)$, and $\beta$ $=(-0.10,0.00,0.00)$ are also shown. They were included since three (one) valence band maxima (conduction band minimum) occurs at $\alpha_{i}(\beta)$, as one can see from the GGA+TS band structure closeup depicted at the bottom part of Fig. 6 . The two 101 planes shown in Fig. 3(c) are also displayed in Fig. 5.

The GGA+TS electronic band structure of the anhydrous monoclinic L-asparagine crystal is shown in Fig. 6, together with its associated partial density of states (PDOS, see right side panels). Electron energies were gauged to ensure that the highest energy valence band has its maximum at $0 \mathrm{eV}$. At the top of Fig. 6, one can see the full energy range of the calculated electron energies, from $-22.50 \mathrm{eV}$ to $12.00 \mathrm{eV}$, with the right panel depicting the contribution from the side chain and carboxyl group to each band. At the bottom, a closeup near the

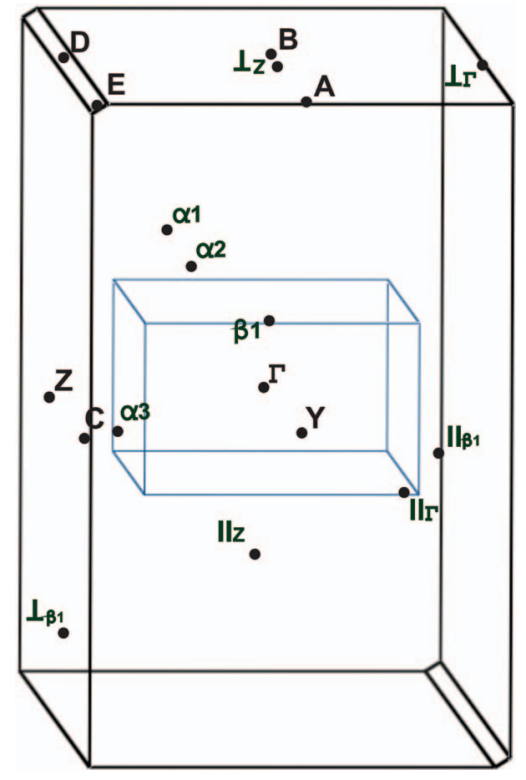

FIG. 5. First Brillouin zone of anhydrous monoclinic asparagine crystals. High symmetry points (latin type, except for $\Gamma$ ) and low symmetry points (greek type symbols, except for $\Gamma$ ) are indicated.
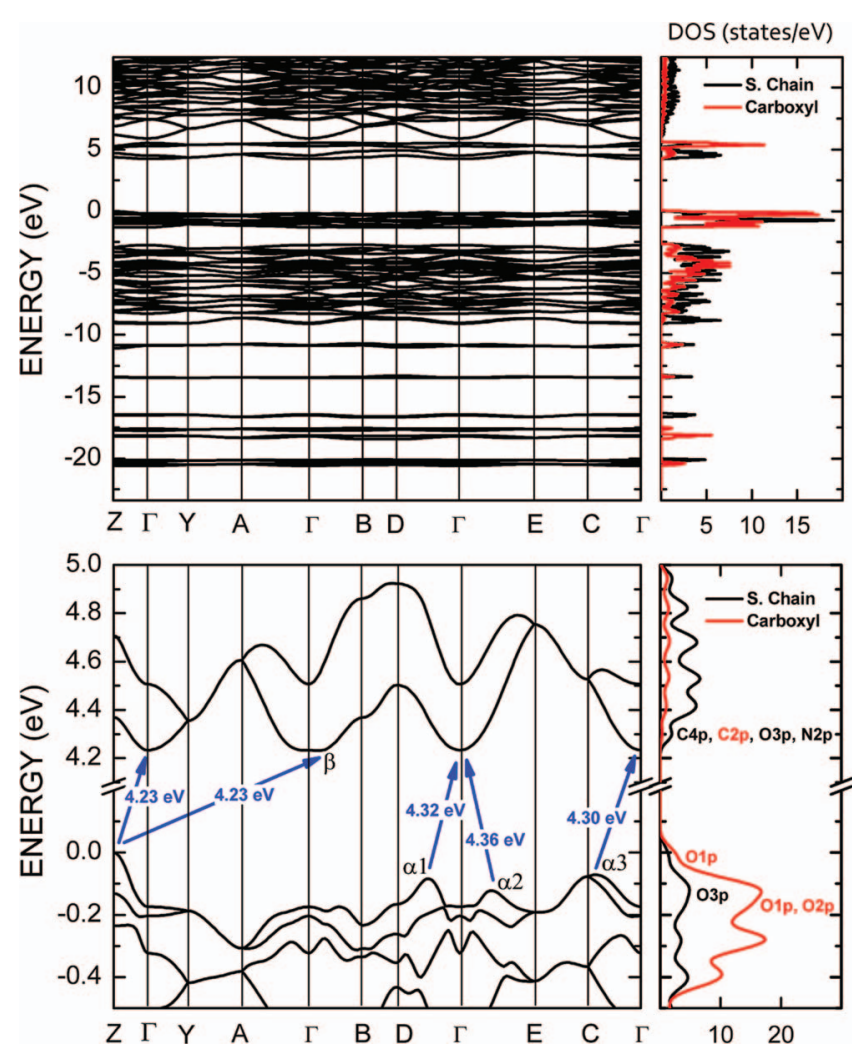

FIG. 6. The GGA+TS calculated band structure of anhydrous monoclinic asparagine crystals. Top: all bands in the -22.5 to $12.5 \mathrm{eV}$ range; bottom: closeup of the band structure around the region of the main energy band gap. The partial density of states displaying the contributions of the side chain (black lines) and carboxyl group (red lines) is shown at the right side panels. The most important atomic orbitals contributing to the highest valence and lowest conduction bands are also specified at some PDOS maxima. 


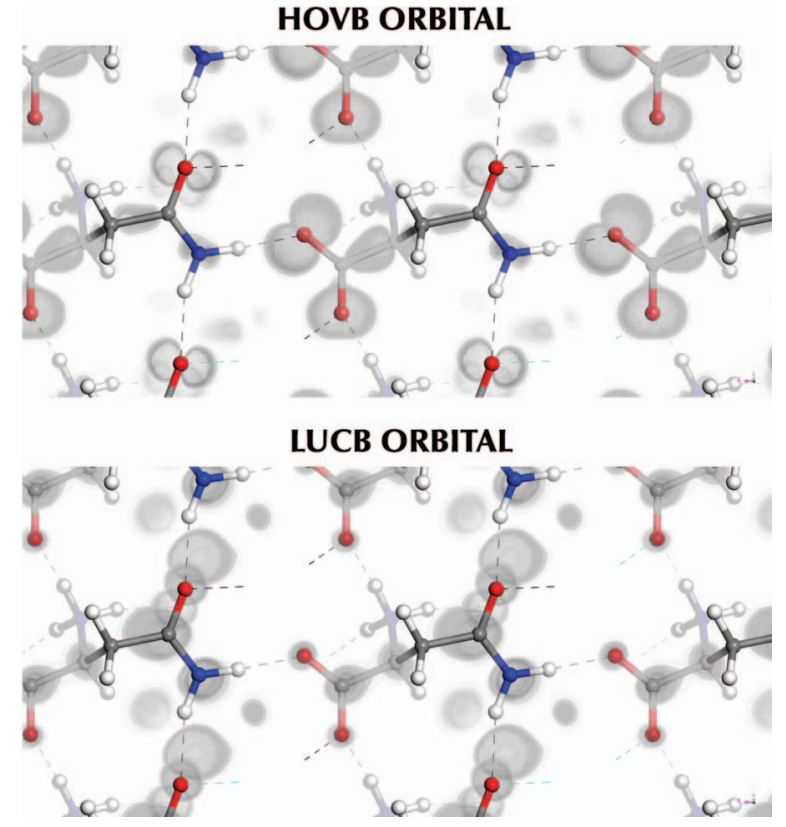

FIG. 7. GGA+TS calculated highest occupied valence band (HOVB) and lowest unoccupied conduction band (LUCB) orbitals.

main band gap reveals the most relevant electronic transitions between the top of the valence band and the bottom of the conduction band, while the partial density of states plot displays the main atomic contributions to each band near the main energy gap. From the band structure curves and DOS plots, we infer that the conduction bands with minima at about 4.30 , 5.18 , and $6.00 \mathrm{eV}$ are mainly related to $\mathrm{C} 4 / \mathrm{O} 3 / \mathrm{N} 2, \mathrm{C} 2 / \mathrm{O} 1 / \mathrm{O} 2$, and $\mathrm{C} 4 / \mathrm{C} 3 / \mathrm{C} 1 p$ orbitals, respectively, in decreasing order of contribution.

On the other hand, the highest energy valence bands are within the energy range between $-1.22 \mathrm{eV}$ and $0 \mathrm{eV}$, the two top valence band curves originating mainly from $\mathrm{O} 1 / \mathrm{O} 3 / \mathrm{O} 2$ $p$ orbitals. The plots of the highest occupied valence band and the lowest unoccupied conduction band electronic states (HOVB and LUCB orbitals, respectively) shown in Fig. 7 are useful to visualize these contributions. One can see that the HOVB orbital is really $p$-like and located mainly at the oxygen atoms $\mathrm{O} 1, \mathrm{O} 2, \mathrm{O} 3$ and along the $\mathrm{C} 1-\mathrm{C} 2$ and $\mathrm{C} 3-\mathrm{C} 4$ bonds (not very pronounced in the PDOS curve), having also small orbital localizations near the $\mathrm{N} 2$ and $\mathrm{N} 1$ atoms. The LUCB orbital, on the other hand, is mainly localized around the $\mathrm{C} 2$, $\mathrm{C} 1$, and $\mathrm{C} 4$ atoms ( $s p$-like orbital shapes), but also with significant orbital lobes at $\mathrm{N} 2$ and $\mathrm{O} 3$ ( $s p$-like orbital shapes), and a small contribution from oxygen atoms $\mathrm{O} 1$ and $\mathrm{O} 2(s-$ like character).

Returning now to the top part of Fig. 6, one can see that, in the -8.30 to $-2.35 \mathrm{eV}$ energy range there is a wide set of bands which is due to $\mathrm{C} 3 / \mathrm{O} 3 / \mathrm{O} 1 p$ orbitals, while near $-9.00 \mathrm{eV}$ there are two bands with strong contribution from the side chain; between -20.8 and $-11.0 \mathrm{eV}$ there are seven sets of flat bands, with those close to $-11.0 \mathrm{eV}$ being due to $\mathrm{C} 3 / \mathrm{C} 1 / \mathrm{C} 4 s$ and $\mathrm{C} 2 / \mathrm{N} 1 / \mathrm{C} 4 p$ orbitals. The bands at about $-13.4 \mathrm{eV}$ originate from $\mathrm{C} 3 / \mathrm{C} 1 / \mathrm{N} 1$ s orbitals, while the bands at $-16.50 \mathrm{eV}$ are due to $\mathrm{N} 2 / \mathrm{O} 3 / \mathrm{C} 4 s$ states. The band sets at $-17.7 \mathrm{eV}$ and $-18.2 \mathrm{eV}$, on the other hand, originate mainly from N1/C1/O1/O2 $s$ orbitals and O2/O $1 s$ and $\mathrm{C} 2$ $p$ orbitals, respectively. Finally, the band sets near $-20.20 \mathrm{eV}$ and $-20.50 \mathrm{eV}$ have strong contributions from O3/C4 $s$ levels and $\mathrm{O} 1 / \mathrm{O} 2 / \mathrm{C} 2 s$ levels, in this order. Overall, the top valence bands are dominated by $p$ states, while the valence bands with lowest energies are mainly originated from $s$ states. The bottom of the conduction bands is strongly $p$-like in character, but the band curves above result from the hybridization of $s$ and $p$ states.

The closeup of the GGA+TS calculated Kohn-Sham band structure near the main band gaps (bottom part of Fig. 6) shows five indirect energy gaps. The smallest gap values are both equal to $4.23 \mathrm{eV}$, corresponding to the valence bandconduction band transitions $\mathrm{Z} \rightarrow \Gamma$ and $\mathrm{Z} \rightarrow \beta$. Secondary indirect band gaps of $4.30 \mathrm{eV}, 4.32 \mathrm{eV}$, and $4.36 \mathrm{eV}$ are related to the transitions $\alpha 3 \rightarrow \Gamma, \alpha 1 \rightarrow \Gamma$, and $\alpha 2 \rightarrow \Gamma$, respectively (the $\alpha_{i}$ and $\beta$ points are depicted in the first Brillouin zone shown in Fig. 5). All the GGA+TS estimated indirect band gap energies, which are in the $4.23-4.36 \mathrm{eV}$ range, are well below the experimental gap of $4.95 \mathrm{eV}$ estimated from our optical absorption measurements. This difference was already expected as pure DFT functionals are not able to predict excitation energies. ${ }^{59-63}$ Similar underestimations of the main band gap by DFT computations were previously reported for several molecular crystals, including amino acid ${ }^{19-22}$ and DNA nucleobases ${ }^{23}$ crystals. Recently, however, a nonempirical scaling correction method for molecules and solids was proposed to correct this gap error. ${ }^{64}$ After applying the $\Delta$-sol correction, ${ }^{55}$ however, the band gap value we obtained was $5.00 \mathrm{eV}$, in much better agreement with the experimental estimate from the optical absorption data (only $50 \mathrm{meV}$ larger).

It is interesting to compare the optical absorption estimated gap $E_{g}^{\exp }=4.95 \mathrm{eV}$, as well as the theoretically calculated indirect gap energy $\mathrm{E}_{g}^{G G A+T S}(\mathrm{Z} \rightarrow \Gamma, \beta)=4.23 \mathrm{eV}$ of the anhydrous monoclinic L-asparagine crystal obtained in this work with similar data for other amino acid crystals. The pioneer work of Tulip and Clark ${ }^{17}$ estimated that alanine orthorhombic crystals and leucine, isoleucine, and valine monoclinic crystals have energy gaps $E_{g} \sim 5.0 \mathrm{eV}$, but details about the types of gap (direct or indirect) and the electronic transitions involved were not provided. A DFT simulation study of monoclinic $\alpha$-glycine crystals $^{19}$ revealed the presence of three close indirect energy gaps, with $\mathrm{E}_{g}^{G G A}=4.95 \mathrm{eV} .{ }^{19}$ On the other hand, both monoclinic and orthorhombic cysteine crystals were predicted to have indirect energy gaps, ${ }^{20}$ the former with an electronic transition $\mathrm{D} \rightarrow \mathrm{E}$ corresponding to an energy gap $\mathrm{E}_{g}^{G G A}=4.06 \mathrm{eV}$ and a secondary transition $\mathrm{A} \rightarrow \mathrm{B}$ with $\mathrm{E}_{g}^{G G A}=4.08 \mathrm{eV}$, while the latter has three electronic transitions $\mathrm{Z} \rightarrow \mathrm{T}, \mathrm{T} \rightarrow \mathrm{Y}$, and $\Gamma \rightarrow \mathrm{Z}$ with practically the same energy $\mathrm{E}_{g}^{G G A}=4.52 \mathrm{eV}$. The lowest energy gap of the anhydrous monoclinic L-aspartic acid crystal was estimated to be $\mathrm{E}_{g}^{G G A+T S}=4.49 \mathrm{eV},{ }^{21}$ being assigned to the indirect transition $\mathrm{B} \rightarrow \alpha_{1}$. The energy gap of the anhydrous monoclinic L-serine crystal calculated using the GGA+TS methodology ${ }^{22}$ was $\mathrm{E}_{g}^{G G A+T S}=4.75 \mathrm{eV}$ due to a $\mathrm{S} \rightarrow \Gamma$ indirect transition.

The experimental gaps for the following amino acid crystals can be found in the literature from optical absorption measurements: L-alanine orthorhombic, $E_{g}^{\text {exp }}=5.27 \mathrm{eV} ;{ }^{65}$ 
leucine, $E_{g}^{\text {exp }}=5.29 \mathrm{eV} ;{ }^{66}$ valine, $E_{g}^{\text {exp }}=5.04 \mathrm{eV} ;{ }^{67}$ glycine, $5.11 \pm 0.02 \mathrm{eV} ;{ }^{19}$ cysteine orthorhombic, $E_{g}^{\exp }=4.68 \mathrm{eV}$ (the gap of monoclinic cysteine was not measured yet) ${ }^{20}$ L-aspartic acid monoclinic, $E_{g}^{\exp }=5.02 \mathrm{eV} ;^{21}$ and L-serine monoclinic, $E_{g}^{\text {exp }}=5.90 \mathrm{eV}{ }^{22}$ The theoretically calculated energy gaps of these amino acids can be ordered by increasing values (in eV) as follows: Cys (4.06, 4.52) < Asn (4.23) $<$ Asp (4.49) < Ser (4.75) < Gly (4.95) < Ala, Leu, Ile, Val (5.0), while the experimental gaps obey the sequence (energies in eV) Cys (4.68) < Asn (4.95) < Asp (5.02) < Val $(5.04)<$ Gly $(5.11)<$ Ala (5.27) < Leu (5.29) < Ser (5.90). There are two disagreements between these sequences involving the L-serine crystal (largest experimental gap, calculated gap smaller than those for Gly, Ala, Leu, Ile, and Val), followed by valine (experimental gap larger than the gap for Gly, while the theoretical gap is smaller).

With the purpose to better understand the relationship between the band gaps of these amino acid crystals and the electronic structure of each amino acid molecule, we have also performed DFT calculations for the isolated molecules asparagine, aspartic acid, histidine, proline, and tryptophan using the GGA+TS exchange-correlation functional. These molecules were chosen in such a way to produce a sample representing the different types of side chains observed in essential amino acids. Two molecular states were assessed: the neutral state in vacuum and the zwitterionic state in water (except for proline, which has its amine bound to two alkyl groups and does not form a zwitterion when solvated), the latter using a continuous solvation scheme. After carrying out a geometry optimization procedure similar to the one used for the L-asparagine crystal, we have obtained the highest occupied molecular orbital (HOMO) and the lowest unoccupied molecular orbital (LUMO) for each molecular configuration, both shown in Fig. 8. Each optimized geometry has, in general, a set of stabilizing internal hydrogen bonds: asparagine has two, aspartic acid has two in vacuum and one in the zwitterion state, histidine has two, proline has none, and tryptophan has a single internal hydrogen bond.

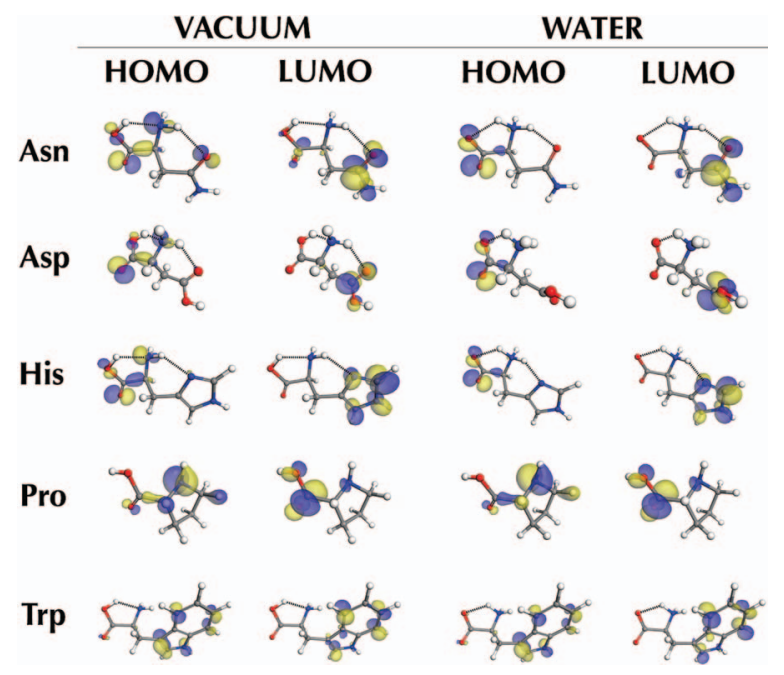

FIG. 8. GGA+TS calculated HOMO and LUMO orbitals for asparagine (Asn), aspartic acid (Asp), histidine (His), proline (Pro), and tryptophan (Trp) in vacuum (left) and solvated in water (right).
The HOMO and LUMO orbitals for asparagine in vacuum are remarkably similar to the HOVB and LUCB orbitals presented in Fig. 7, notwithstanding the fact that the crystal is formed from molecules in the zwitterionic state. This seems to indicate that the insertion of water molecules in the crystalline phase will affect significantly the electronic structure in comparison with the anhydrous crystal. The HOMO and LUMO for zwitterionic aspartic acid differ from the vacuum case mainly by the absence of a significant contribution from the ammonium group (amine protonated) to the HOMO and from the carboxyl group to the LUMO. For the aspartic acid molecule in vacuum, the HOMO orbital is mainly located at the carboxyl group, while the LUMO is concentrated at the side chain. As for the zwitterion aspartic acid (as in the case of asparagine), the ammonium contribution to the HOMO disappears (and the same occurs for histidine), while the carboxyl side chain is twisted relative to the vacuum geometry. Histidine has its two LUMO orbitals (in vacuum and solvated in water) located at its side chain, and its two HOMO orbitals located mainly at the carboxyl and deprotonated carboxyl regions. Proline, on the other hand, barely changes its HOMO and LUMO shapes as we switch from vacuum to water; tryptophan has both its HOMO and LUMO states located at the indole side chain.

The HOMO-LUMO energy gaps calculated for the amino acid molecules in vacuum were, in crescent order: $3.67 \mathrm{eV}$ for tryptophan, $4.03 \mathrm{eV}$ for proline, $4.24 \mathrm{eV}$ for aspartic acid, $4.61 \mathrm{eV}$ for asparagine, and $4.76 \mathrm{eV}$ for histidine. When they are solvated in water, their gap figures change to $3.63 \mathrm{eV}$ for tryptophan, $3.97 \mathrm{eV}$ for proline, $4.33 \mathrm{eV}$ for aspartic acid, $4.55 \mathrm{eV}$ for asparagine, and $5.00 \mathrm{eV}$ for histidine. In the case of tryptophan, which has the smallest band gap, both the HOMO and LUMO orbitals are located at the side chain (Fig. 8), which must, in principle, lead to larger band dispersion in tryptophan crystals. The highest HOMO-LUMO gap for histidine, by the way, seems to be related with the small degree of overlap between the HOMO and LUMO orbitals for this amino acid, which also occurs to asparagine and aspartic acid. Proline, on the other hand, exhibits HOMO and LUMO orbitals with some degree of overlap, leading to the second smallest HOMO-LUMO band gap. Besides, it also has a very small variation in the HOMO-LUMO gap as we switch from vacuum to water, as their frontier orbitals do not change significantly with solvation. It is also worth to note that asparagine and aspartic acid crystals have very close experimental band gaps, while their DFT-calculated estimates predict that aspartic acid crystals have an energy gap $0.26 \mathrm{eV}$ larger than the value for asparagine crystals, a trend which reverses the order of molecular HOMO-LUMO gaps. Thus, as a description based only in the analysis of the isolated molecules is clearly not enough to explain the experimentally observed gap differences, one must conclude that intermolecular interaction effects cannot be ignored in order to explain the electronic properties of amino acid crystals.

Effective masses for electrons and holes can be estimated from band curvatures at valence band maxima and conduction band minima, being useful to estimate the mobility of the charge carriers in a given direction and also to evaluate if the intermolecular interactions in the crystal are strong enough to 

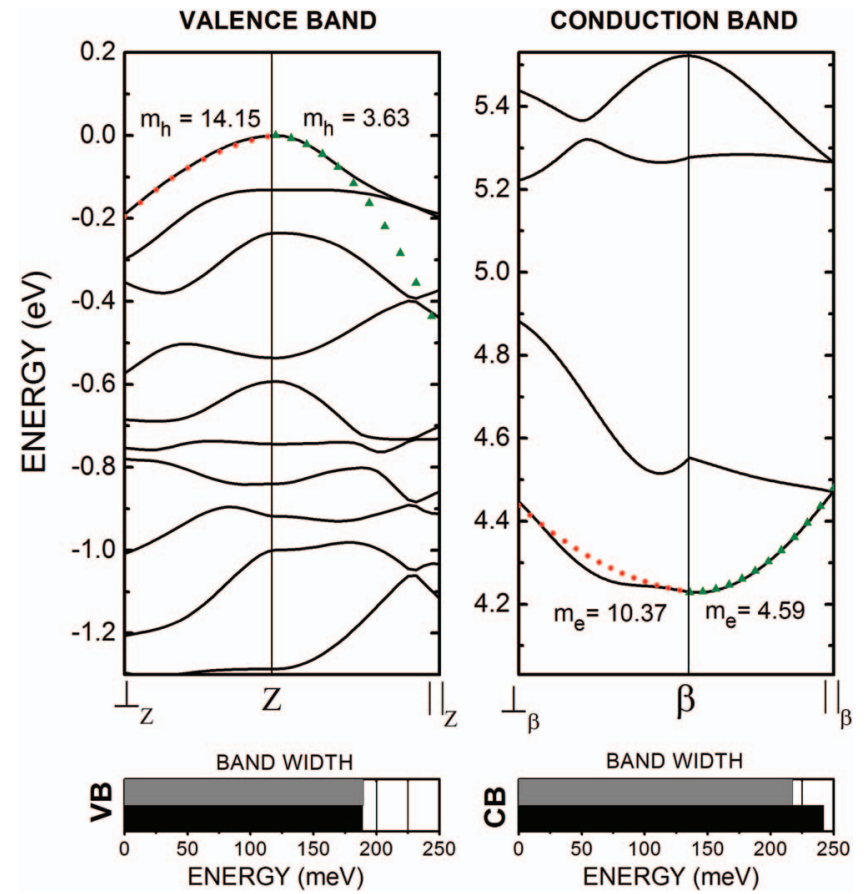

FIG. 9. GGA+TS estimated electron and hole effective masses (in free electron mass units, $m_{o}$ ) for the anhydrous monoclinic asparagine crystal (above), and valence VB and conduction band $\mathrm{CB}$ widths (below). The fittings were performed at the valence maximum and conduction band minimum along directions parallel and perpendicular to the molecular layers of asparagine, as shown in Fig. 5. Gray (black) band widths correspond to the band along the parallel (perpendicular) direction.

affect the electronic structure and produce a significant difference with respect to pure molecular states. The effective mass calculation procedure is easy to implement: one must take the inverse of the second derivative of the energy with respect to the wavevector at a critical point in the first Brillouin zone and project it along a chosen path. As a rule of thumb, one can say that the larger (smaller) the band width near the critical point, the smaller (larger) the effective mass will be. For the anhydrous asparagine crystal, we have first evaluated the effective masses for the uppermost valence band, centered at the $\mathrm{Z}$ point (hole effective masses) and for the lowermost conduction band, centered at the $\beta$ point, which is situated along the linear path connecting the $\Gamma$ and B points (see bottom of Fig. 6). Two directions were selected for each point: parallel $(\|)$ and perpendicular $(\perp)$ to the molecular planes of asparagine along the 101 direction (see Fig. 3(c)). The values obtained, together with the band widths in the neighborhood of these points, are shown in Fig. 9. For the perpendicular directions, we see that the hole and electron effective masses are larger than $10 \mathrm{~m}_{0}$, where $\mathrm{m}_{0}$ is the free electron masses, which points to the difficulty of moving the charge carriers from one molecular layer to the next. For electrons, on the other hand, the band curves have larger dispersion, leading to effective masses of about $3.63 \mathrm{~m}_{0}\left(\mathrm{Z}-\|_{\mathrm{Z}}\right)$ and $4.59 \mathrm{~m}_{0}$ $\left(\beta-\|_{\beta}\right)$, which suggests that a wide gap semiconductor behavior can be achieved for charge carriers moving within these 101 molecular layers. Looking just to the band widths, one cannot predict this result, however, as the bands for both

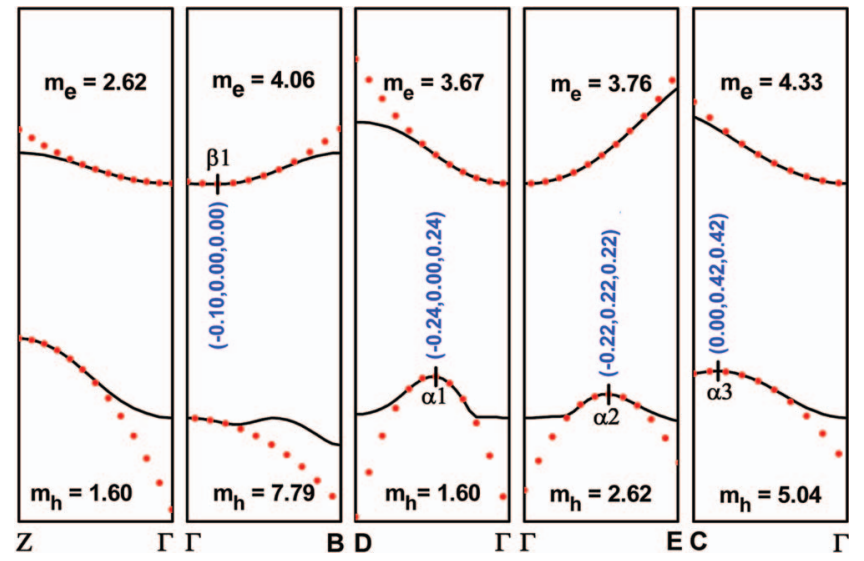

FIG. 10. GGA+TS estimated electron and hole effective masses (free electron mass units) for the anhydrous monoclinic asparagine crystal. Parabolic fittings are also shown.

parallel and perpendicular effective masses have nearly the same range of energy variation.

Figure 10 presents five panels with the uppermost (lowermost) valence (conduction) bands, each one starting or ending at the $\Gamma$ point along the $k$-axis, with corresponding hole (electron) effective masses. The valence band maxima $\alpha_{i}$ and the conduction band minimum $\beta$ are indicated, and effective masses were obtained for them along a selected set of directions. For holes, there is a very strong anisotropy in effective mass values, ranging from $1.60 \mathrm{~m}_{0}$ (along the $\mathrm{Z}-\Gamma$ and $\mathrm{D}-\alpha_{1}-\Gamma$ paths) to $7.79 \mathrm{~m}_{0}$ (along $\Gamma-\mathrm{B}$ ). In the case of electrons, however, there is a smaller degree of variation, with the largest effective mass $\left(4.33 \mathrm{~m}_{0}\right)$ being observed at $\Gamma$ for the $\Gamma-\mathrm{C}$ direction and the smallest one $\left(2.62 \mathrm{~m}_{0}\right)$ at $\Gamma$ along $\Gamma-Z$ Z. Notwithstanding the fact that the calculated effective masses are somewhat large (always larger than the free electron mass), they are always mensurable, and some dispersion of the band curves, although not as large as it occurs for covalent inorganic crystals, indicates that the interaction between asparagine molecules in the monoclinic phase is strong enough to allow for the usefulness of the electronic band structure picture.

Additional details on the atomic and functional group contributions to the density of electronic states can be seen in Figs. 11 and 12. The per orbital per atom partial density of states, depicting the relative contributions of all carbon, oxygen, nitrogen, and hydrogen atoms to the total DOS of the anhydrous asparagine crystals is shown in Fig. 11. As one can see from it, $\mathrm{O} 2 p$ gives the most significant contribution to the top of the valence band, while the $\mathrm{C} 2 p$ levels originate most part of the bottom of the conduction band. $\mathrm{H} 1 s$ states, on the other hand, contribute significantly to the conduction bands above $6.2 \mathrm{eV}$, as well as the $\mathrm{C} 2 s, \mathrm{C} 2 p$, and $\mathrm{N} 2 p$ ones. Valence bands between $-5.0 \mathrm{eV}$ and $-2.4 \mathrm{eV}$ are dominated by $\mathrm{C} 2 p$ states above $-3.7 \mathrm{eV}$ and by $\mathrm{O} 2 p$ states below, while the bands between $-9.0 \mathrm{eV}$ and $-5.0 \mathrm{eV}$ are mainly due to the $\mathrm{N} 2 p$ orbitals.

Looking now to Fig. 12, one can see that the top valence band has a strong contribution from both the oxygens at the carboxyl group and side chain, while the $\mathrm{H} 1 s$ 


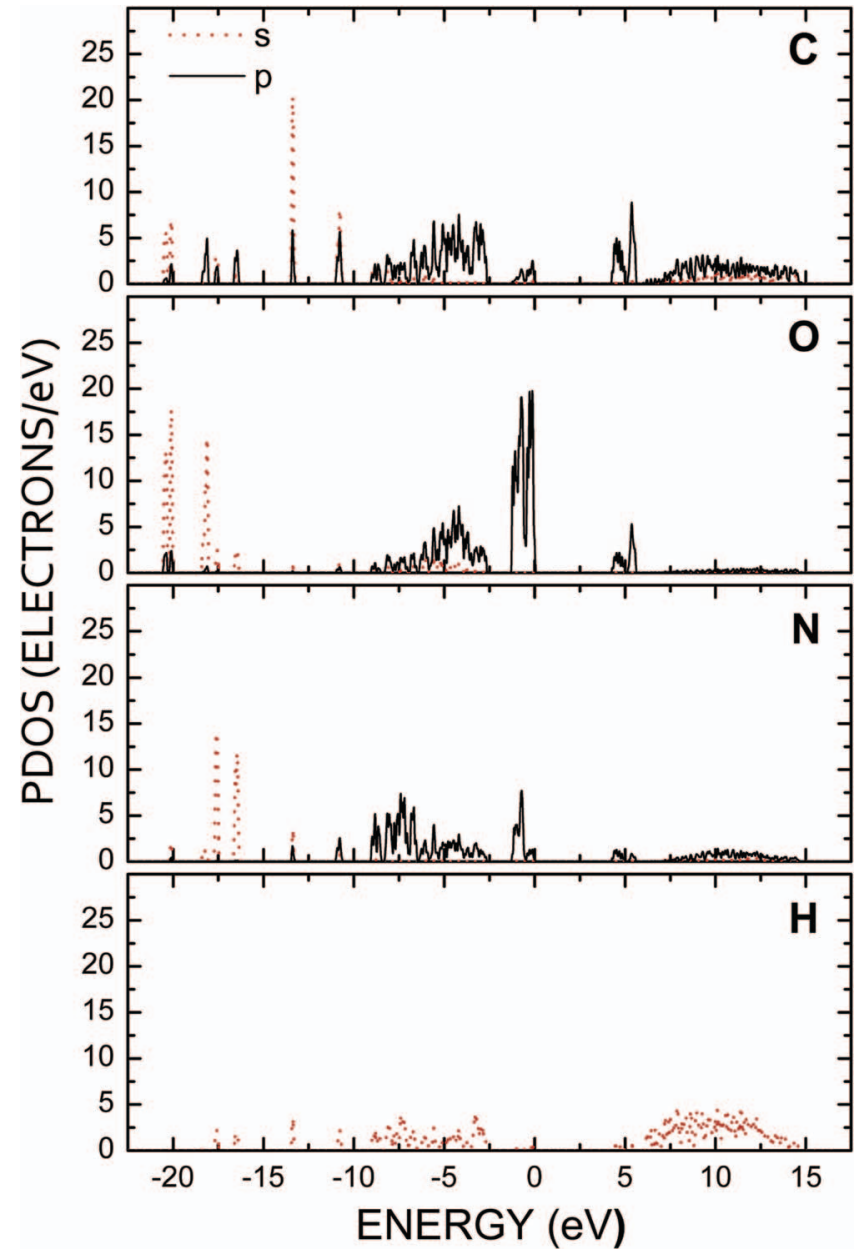

FIG. 11. Anhydrous asparagine monoclinic crystal GGA+TS partial densities of states for $\mathrm{C}, \mathrm{O}, \mathrm{N}$, and $\mathrm{H}$ : $s$ (dotted, red) and $p$ (solid, black) orbitals.

contribution for the conduction bands above $6.0 \mathrm{eV}$ comes from the side chain, followed by the amine group. The bottom of the conduction band, on the other hand, originates from the carbon atoms at the side chain and, for energies a bit larger (about $5.0 \mathrm{eV}$ ), from the carbon atoms at the carboxyl group. A set of $\mathrm{O} 2 s, \mathrm{~N} 2 s$, and $\mathrm{H} 1 \mathrm{~s}$ deep valence levels can be assigned to the carboxyl group near $-20.4 \mathrm{eV}$ and $-18.0 \mathrm{eV}$ (mainly oxygen), to the amine group at $-17.5 \mathrm{eV}$ (mainly nitrogen), and to the side chain at $-20.1 \mathrm{eV}$ (mainly oxygen), $-16.4 \mathrm{eV}$ (nitrogen), and $-13.3 \mathrm{eV}$ (carbon).

One can infer from these results that the main indirect gap transition occurs between a valence electronic state with localization at the carboxyl and side chain and a conduction state located mostly at the side chain, with a smaller contribution from the carboxyl region, as can also be seen from Fig. 7. More details on the specific contributions of each atom of asparagine can be found in the supplementary material (Figs. 1-8 therein). ${ }^{58}$

Intermolecular interactions, charged states, and charge transfer in amino acid crystals can play a role in the unlocking of amino acid molecules from internal hydrogen bonds. Consequently, it is interesting to estimate how changes in the hydrogen bond patterns can influence the electrical charge of each atom. Among several methods of charge partition-

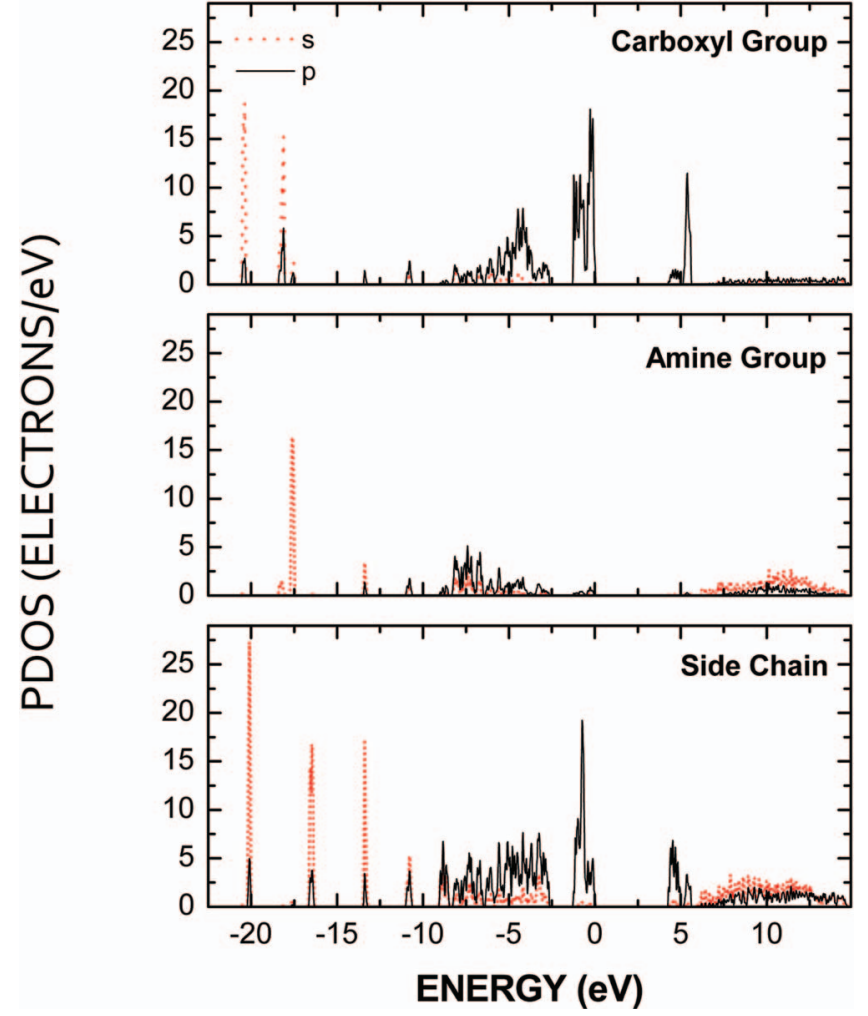

FIG. 12. Anhydrous asparagine monoclinic crystal GGA+TS partial densities of states for the carboxyl, $\mathrm{COOH}$ lateral chain, and amine groups: $s$ (dotted, red) and $p$ (solid, black) orbitals.

ing available in the literature, Hirshfeld population analysis (HPA) ${ }^{68}$ provides one of the most promising approaches, as we have pointed out in another paper. ${ }^{21}$ HPA produces improved Fukui function indices ${ }^{69-71}$ capable of predicting reactivity trends within a molecule better than Mulliken population analysis, ${ }^{72}$ natural bond orbital analysis, ${ }^{73}$ and fitted electrostatic potentials. ${ }^{74}$ Besides, HPA minimizes the loss of information related to the formation of chemical bonds between atoms in a molecule. ${ }^{75,76}$ However, its values tend to be too small, ${ }^{77,78}$ as Hirshfeld atoms in general resemble the neutral atoms. ${ }^{79,80}$ This limitation, however, can be amended using the iterative Hirshfeld charge technique, ${ }^{78}$ which has been successfully applied to the solid state ${ }^{81}$ and the discussion of Fukui functions. ${ }^{82}$

In Table II, we present the GGA+TS Hirshfeld charges (in units of the fundamental charge $e$ ) of the individual atoms

TABLE II. Hirshfeld atomic charges (in $e$ units) for the L-asparagine molecule in the anhydrous monoclinic Asn crystal as calculated at the GGA+TS level.

\begin{tabular}{lrrrrr}
\hline \hline Atom & $\mathrm{H}_{G G A+T S}$ & Atom & $\mathrm{H}_{G G A+T S}$ & Atom & $\mathrm{H}_{G G A+T S}$ \\
\hline $\mathrm{C} 1$ & 0.02 & $\mathrm{~N} 1$ & -0.07 & $\mathrm{H} 6$ & 0.11 \\
$\mathrm{C} 2$ & 0.16 & $\mathrm{~N} 2$ & -0.15 & $\mathrm{H} 7$ & 0.10 \\
$\mathrm{C} 3$ & -0.08 & $\mathrm{H} 1$ & 0.03 & $\mathrm{H} 8$ & 0.10 \\
$\mathrm{C} 4$ & 0.17 & $\mathrm{H} 2$ & 0.09 & Carb. & -0.29 \\
$\mathrm{O} 1$ & -0.23 & $\mathrm{H} 3$ & 0.10 & Am. & 0.24 \\
$\mathrm{O} 2$ & -0.22 & $\mathrm{H} 4$ & 0.04 & S.C. & -0.01 \\
$\mathrm{O} 3$ & -0.22 & $\mathrm{H} 5$ & 0.04 & $\ldots$ & $\ldots$ \\
\hline \hline
\end{tabular}


and functional groups (carboxyl, amine, side chain) in a single asparagine molecule belonging to the anhydrous Asn monoclinic crystalline phase. They were calculated using the HPA scheme available in the CASTEP code. ${ }^{40}$ One can see that $\mathrm{O} 1, \mathrm{O} 2, \mathrm{O} 3$ are the most negatively charged atomic species (Hirshfeld charges of $-0.23,-0.22$, and $-0.22 e$, respectively). The $\mathrm{N} 2$ nitrogen atom belonging to the side chain is negatively charged $(-0.15 e)$, as well as the N1 at the amine group, which exhibits a small negative Hirshfeld atomic charge $(-0.07 e$, like the $\mathrm{C} 3$ atom of the side chain, with $-0.08 e$ ). The carboxyl, amine, and side chain groups have Hirshfeld GGA+TS calculated charges of -0.29 $e, 0.24 e$, and $-0.01 e$, respectively, which reveals the dipolar character of the zwitterionic L-asparagine molecules in the anhydrous monoclinic crystal.

One can argue that intermolecular interactions involving hydrogen bonds are somehow responsible for the small negative charge of the side chain of L-asparagine molecules in anhydrous monoclinic crystals. As a matter of fact, if we suppose that charged side chains are also present in all amino acid crystals as it occurs for the isolated amino acid species and for polypeptides interacting in aqueous environment, then it seems reasonable to consider that the folded state of proteins (or their structure-activity relationship) is related to charge transfer processes occurring at their side chains, which are affected by the particular combination of hydrogen bonds and van der Waals forces related to their peptidic backbone. Besides, there is a relationship between charge redistribution processes due to intermolecular interactions and the complex pattern of the spatially dependent complex function of proteins. ${ }^{83-86}$ It is not less important to associate changes in the local $\mathrm{pK}_{a}$ of amino acids in proteins to the charge transfer, affected by the hydrogen bond pattern, at their side chains. Within this picture, the crystal field connection with the asparagine hydrogen bond unlocking process (see Fig. 1), together with the charge transfer to the asparagine side chain as obtained by the GGA+TS calculations (see Table II), suggest the important role of hydrogen bonds on the protein folding route and on the inhomogeneous dielectric function. However, further studies are necessary to fully unveil the role of hydrogen bond unlocking in proteins. Under such viewpoint, the study of the structural and electronic properties of amino acid crystals is the first step to be taken. ${ }^{18-22}$

\section{OPTICAL PROPERTIES}

In previous works, the DFT calculated energy band gaps for monoclinic $\alpha$-glycine ${ }^{19}$ and orthorhombic cysteine ${ }^{20}$ were close to the values obtained from optical absorption measurements. For the $\alpha$-glycine crystal, we have found $E_{\text {exp }}=5.11 \mathrm{eV}$ and an indirect DFT gap $E_{\mathrm{DFT}}=4.89 \mathrm{eV}$, a difference of $0.22 \mathrm{eV}$ (about $-4 \%$ ). For cysteine, $E_{\text {exp }}=4.68 \mathrm{eV}$ and the smallest calculated gap was indirect, $E_{\mathrm{DFT}}=4.52 \mathrm{eV}$, the theoretical estimate being smaller than experiment by only $0.16 \mathrm{eV}$ (about $-3 \%$ ). On the other hand, for monoclinic anhydrous L-aspartic acid crystals we have obtained $E_{\text {exp }}=5.02 \mathrm{eV}$ and an indirect gap $E_{\mathrm{DFT}}=4.49 \mathrm{eV}$ $(0.53 \mathrm{eV}$ or $-11 \%$ smaller than experiment), while for anhydrous monoclinic L-serine crystals, $E_{\exp }=5.11 \mathrm{eV}$, with

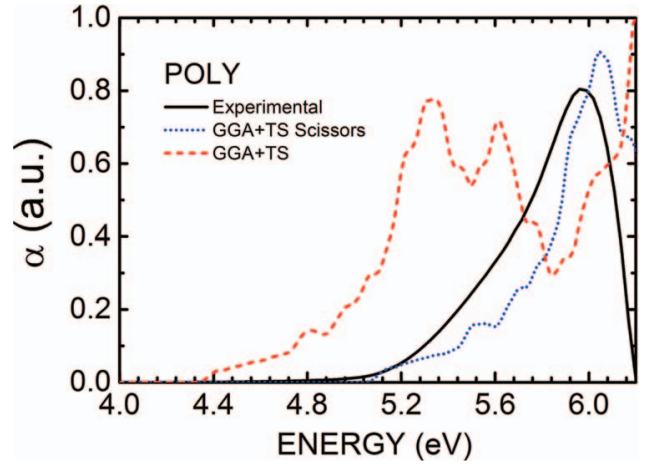

FIG. 13. Comparison between the optical absorption of the anhydrous monoclinic asparagine measured with nonpolarized incident radiation at $300 \mathrm{~K}$ (solid line) and the GGA+TS calculated optical absorption for a polycrystalline sample with scissor shift (blue dotted line) and without scissor shift (red dashed line).

DFT predicting a direct gap $E_{\mathrm{DFT}}=4.74 \mathrm{eV}(0.37 \mathrm{eV}$ or $-7 \%$ smaller than experiment). Considering that DFT calculations typically underestimate band gaps by $50 \%$, our calculated figures are surprisingly close to the experimental data. For the L-asparagine crystal, this trend is also verified as the optical measurements pointed to a band gap of $4.95 \mathrm{eV}$ while the GGA+TS computations predicted a minimal indirect band gap of $4.23 \mathrm{eV}$, a difference of $0.72 \mathrm{eV}$ or $-15 \%$. Taking into account this difference, we have calculated the optical properties (absorption, dielectric function) for the case of incident light with polarization and for a polycrystalline sample using a scissors operator to shift up the theoretical band gap by $0.72 \mathrm{eV}$ (the shift for the $\Delta$-sol estimate would be almost the same, $0.77 \mathrm{eV}$ ). The optical absorption curves with and without the scissor operator in the case of a polycrystal are shown in Fig. 13, together with the experimental result. As one can see, the GGA+TS scissor curve is in good agreement with the measured data.

Figure 14 depicts the optical absorption obtained from the GGA+TS scissors electronic structure in the case of in-

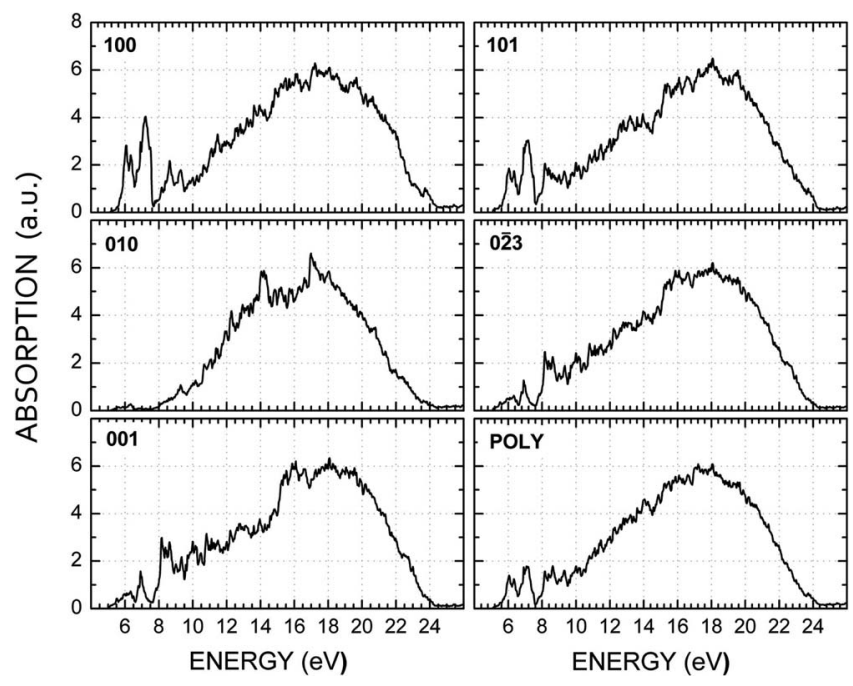

FIG. 14. GGA+TS calculated optical absorption of the anhydrous monoclinic asparagine crystal for light polarized along the directions 100, 010, 001,101 , and $0 \overline{2} 3$. The optical absorption for the polycrystal is also shown. 


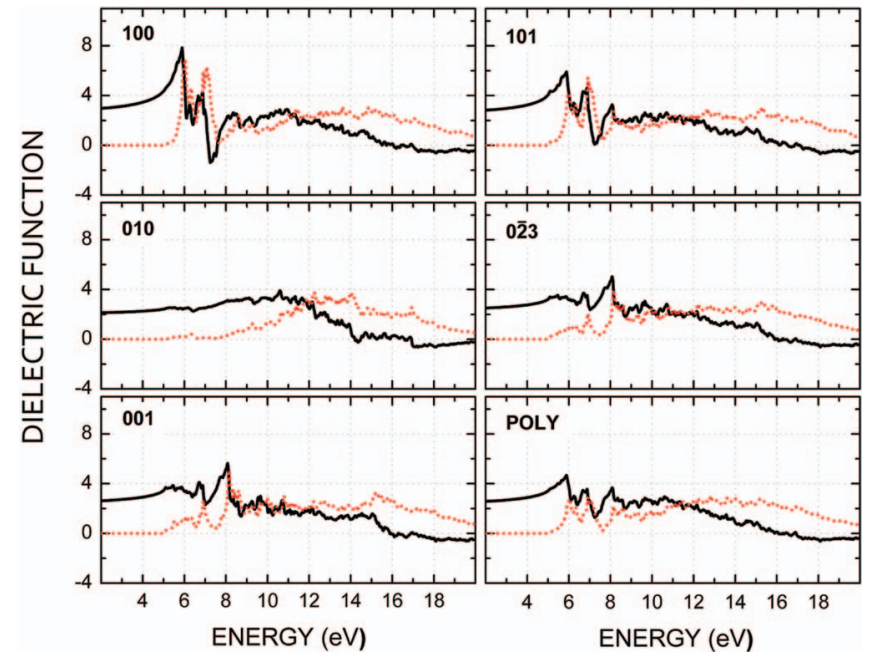

FIG. 15. Real (solid black line) and imaginary (dotted red line) components of the GGA+TS calculated dielectric function of the anhydrous monoclinic asparagine crystal for light polarized along the directions 100, 010, 001, 101 , and $0 \overline{2} 3$. The dielectric function components for the polycrystal are also shown.

cident light polarized along a chosen set of directions (100, 010, 001, 101, and 02̄3). The absorption curves reveal that anhydrous monoclinic L-asparagine crystals are remarkably anisotropic optically. For example, the optical absorption spectrum for polarization along 100 (which is parallel to the crystalline intramolecular tunnels, see Fig. 3(d)), for energies below $8.0 \mathrm{eV}$, displays a set of very pronounced peaks at $6.0 \mathrm{eV}$ and $7.1 \mathrm{eV}$, while for the 001 polarization there is a single absorption peak at nearly $6.9 \mathrm{eV}$, and for 010 polarization the optical absorption is very small in the same energy range. For the 101 direction, which is parallel to the molecular layers of L-asparagine, the optical absorption for smaller energies resembles the 100 curve with some degree of attenuation. In the case of $0 \overline{2} 3$ polarization, which corresponds to the direction of the hydrogen bond $\mathrm{O}_{2} \cdots \mathrm{N}_{1} \mathrm{H}_{7}$ between the amine and carboxyl groups of neighbor asparagine molecules inside the unit cell, the absorption curve below $8.0 \mathrm{eV}$ has a pronounced peak at about $7.0 \mathrm{eV}$ and looks very similar to the absorption curve for the 001 polarization.

Finally, in Fig. 15 we see the GGA+TS calculated real and imaginary parts of the dielectric function, $\epsilon(\omega)=\epsilon_{1}(\omega)$ $+i \epsilon_{2}(\omega)$, following the same scheme of polarization directions of Fig. 14. The imaginary part $\epsilon_{2}$ of the dielectric function is proportional to the optical absorption we have discussed in the previous paragraph, so we focus now on the analysis of the real part $\epsilon_{1}$. As expected, the real part of dielectric function is very polarization sensitive, as occurred for the optical absorption. The dielectric function curves for the 100 and 101 polarization directions are considerably more structured, with a larger number of pronounced maxima and minima in comparison with the 010 case. For the 100 polarization we have a pronounced maximum for $\epsilon_{1}$ at $5.9 \mathrm{eV}$ and a secondary maximum at $6.7 \mathrm{eV}$, followed by a minimum at 7.3 $\mathrm{eV}$. One can note that $\epsilon_{1}$ becomes negative in the $7.1 \mathrm{eV}-7.6$ $\mathrm{eV}$ energy range, and above $16.0 \mathrm{eV}$ (the negative sign means that the molecular induced dipoles are oscillating out of phase with respect to the electric field). In the case of 010 polar- ization, $\epsilon_{1}$ is practically constant (approximately 2.15) in the $2.0-6.5 \mathrm{eV}$ energy range, increases slightly to 3.30 for energies between $6.5 \mathrm{eV}$ and $9.1 \mathrm{eV}$, and then falls off, becoming negative at $14.3 \mathrm{eV}$ and above $16.9 \mathrm{eV}$. The real part of the dielectric function for 001 polarization has its main maximum at $8.1 \mathrm{eV}$, assuming negative values for energies larger than $16.0 \mathrm{eV}$ only. The $\epsilon_{1}$ curve in the 101 polarization plot has a maximum at $5.9 \mathrm{eV}$ and a minimum at $7.3 \mathrm{eV}$, as for the 100 case. Finally, for light polarized along $0 \overline{2} 3, \epsilon_{1}$ looks very similar to the 001 curve, as well as $\epsilon_{1}$ for a polycrystalline sample.

\section{CONCLUSIONS}

The anhydrous monoclinic L-asparagine crystal was studied in this work as a new contribution to obtain a complete picture of the properties of amino acids in the solid state. The optical absorption of its powder was measured and its structural, electronic, and optical properties were computed using the DFT-GGA+TS dispersion corrected exchange-correlation functional. An indirect band gap of $4.95 \mathrm{eV}$ was estimated from the optical absorption data, suggesting that L-asparagine crystals can behave as a wide band gap semiconductor or insulator depending on the effective mass-related electrical mobility of their charge carriers.

As for the DFT-GGA+TS calculations, a convergence study was carried out to ensure the quality of the geometry optimization procedure. The calculated unit cell parameters showed very small deviations in comparison with X-ray diffraction data: $\Delta a=0.020 \AA, \Delta b=0.051 \AA, \Delta c=0.022$ $\AA, \Delta \beta=-0.420^{\circ}$, when a $830 \mathrm{eV}$ cutoff energy for the plane wave basis set was employed. Unit cell parameters calculated using the Grimme dispersion correction scheme were as good as the values obtained using the GGA+TS approach. The unit cell total energy variation as a function of the lattice parameter revealed that the unit cell is less stiff along the $b$ direction.

The electronic band structure, partial density of states, carrier effective masses, Hirshfeld charges, and optical properties (absorption and dielectric function) for the L-asparagine crystal were obtained after the unit cell with minimal total energy was achieved. Its electronic band structure exhibited a $4.23 \mathrm{eV}$ indirect band gap for both the $Z \rightarrow \Gamma, \beta$ transitions, and three close indirect band gaps of $4.30 \mathrm{eV}$ $\left(\alpha_{3} \rightarrow \Gamma\right), 4.32 \mathrm{eV}\left(\alpha_{1} \rightarrow \Gamma\right)$, and $4.36 \mathrm{eV}\left(\alpha_{2} \rightarrow \Gamma\right)$. After applying the $\Delta$-sol gap correction scheme, the main gap increases to $5.00 \mathrm{eV}$, almost matching the experimental gap estimate. The $\mathrm{O} 2 p$ carboxyl and the $\mathrm{C} 4$ side chain, $\mathrm{C} 2 p$ carboxyl orbitals contributed most significantly to the highest valence and lowest conduction energy bands, in this order. A comparison between the HOMO-LUMO gaps between molecular asparagine and other four amino acids (aspartic acid, histidine, proline, and tryptophan) reinforced the need to take into account intermolecular interactions to describe adequately the observed band gap differences among amino acid crystals. In particular, the HOVB and LUCB orbitals in L-asparagine crystals exhibit a structure which resembles more closely the HOMO and LUMO states of L-asparagine in vacuum than those of L-asparagine in water, notwithstanding the fact that only the water solvated form is zwitterionic as in the crystal. 
On the other hand, effective mass values pointed to anisotropic hole effective masses ranging from $1.60 \mathrm{~m}_{0}$ (at the $\alpha_{1}$ point along the $\mathrm{D}-\Gamma$ path) to $14.1 \mathrm{~m}_{0}$ (at $\mathrm{Z}$ along a direction perpendicular to the planes of L-asparagine approximately aligned to the 101 direction) free electron masses. For electrons, the effective mass is more isotropic, varying from $2.62 \mathrm{~m}_{0}$ (at $\Gamma$, along $\Gamma-\mathrm{Z}$ ) up to $10.4 \mathrm{~m}_{0}$ (at $\beta$, along the perpendicular to the L-asparagine 101 planes). From the calculated carrier effective masses, it seems that for most directions the anhydrous monoclinic L-asparagine crystals can behave as a semiconductor, the exception being the transport between parallel molecular planes, which has large effective masses and then suggests an insulator character. From the charge population analysis, it was inferred that the O1, $\mathrm{O} 2$ (carboxyl), and $\mathrm{O} 3$ (side chain) atoms are the most negatively charged, followed by the $\mathrm{N} 2$ atom and the $\mathrm{C} 3$ atom, while the largest positive charge was observed for the $\mathrm{C} 4$ and $\mathrm{C} 2$ atoms (side chain and carboxyl groups), followed by H6, H7, H8 (amine group hydrogens), and H3. Finally, the optical properties (absorption and dielectric function) displayed a very strong anisotropy to the incidence of polarized light, with more structured curves for the 100 and 001 polarization directions.

\section{ACKNOWLEDGMENTS}

V.N.F. and C.G. are senior researchers from the Brazilian National Research Council (CNPq), and would like to acknowledge the financial support received during the development of this work from the Brazilian Research Agency CNPq-INCT-Nano(Bio)Simes Project No. 573925/2008-9. E.W.S.C. received financial support from CNPq Projects Nos. 304283/2010-0 and 474734/2011-0.

${ }^{1}$ J. H. McKerrow and A. B. Robinson, Science 183, 85 (1974).

${ }^{2}$ N. E. Robinson and A. B. Robinson, Proc. Natl. Acad. Sci. U.S.A. 98, 12409 (2001).

${ }^{3}$ E. B. Dunkelberger, L. E. Buchanan, P. Marek, P. Cao, D. P. Raleigh, and M. T. Zanni, J. Am. Chem. Soc. 134, 12658 (2012).

${ }^{4}$ M. Manning, D. Chou, B. Murphy, R. Payne, and D. Katayama, Pharm. Res. 27, 544 (2010).

${ }^{5}$ B. Peters and B. L. Trout, Biochemistry 45, 5384 (2006).

${ }^{6}$ S. Catak, G. Monard, V. Aviyente, and M. F. Ruiz-López, J. Phys. Chem. A 110, 8354 (2006).

${ }^{7}$ S. Catak, G. Monard, V. Aviyente, and M. F. Ruiz-López, J. Phys. Chem. A 112, 8752 (2008).

${ }^{8}$ P. Hao, Y. Ren, A. J. Alpert, and S. K. Sze, Mol. Cellular Proteom. 10, O111.009381 (2011).

${ }^{9}$ M. Chen, Z. Huang, and Z. Lin, J. Mol. Struct.: THEOCHEM 719, 153 (2005).

${ }^{10}$ B. Boeckx and G. Maes, Biophys. Chem. 165-166, 62 (2012).

${ }^{11}$ C. Cabezas, M. Varela, I. Pena, S. Mata, J. C. Lopez, and J. L. Alonso, Chem. Commun. 48, 5934 (2012).

${ }^{12}$ A. R. K. Selvaraj, N. A. Murugan, and H. Ågren, J. Phys. Chem. A 116, 11702 (2012).

${ }^{13}$ K. Yamada, D. Hashizume, T. Shimizu, and S. Yokoyama, Acta Crystallogr. Sec. E 63, o3802 (2007)

${ }^{14}$ G. Kartha and A. De Vries, Nature (London) 192, 862 (1961).

${ }^{15}$ M. Ramanadham, S. K. Sikka, and R. Chidambaram, Acta Crystallogr. Sec. B 28, 3000 (1972).

${ }^{16}$ J. J. Verbist, M. S. Lehmann, T. F. Koetzle, and W. C. Hamilton, Acta Crystallogr. Sec. B 28, 3006 (1972).

${ }^{17}$ P. R. Tulip and S. J. Clark, Phys. Rev. B 71, 195117 (2005).

${ }^{18}$ E. W. S. Caetano, M. Z. S. Flores, G. A. Bezerra, J. R. Pinheiro, V. N. Reire, G. A. Farias, J. R. L. Fernadez, J. R. Leite, M. C. F. De Oliveira, J.
A. Pinheiro, B. S. Cavada, and D. L. De Lima Filho, AIP Conf. Proc. 772, 1095 (2005).

${ }^{19}$ M. Z. S. Flores, V. N. Freire, R. P. dos Santos, G. A. Farias, E. W. S. Caetano, M. C. F. de Oliveira, J. R. L. Fernandez, L. M. R. Scolfaro, M. J. B. Bezerra, T. M. Oliveira, G. A. Bezerra, B. S. Cavada, and H. W. Leite Alves, Phys. Rev. B 77, 115104 (2008).

${ }^{20}$ J. R. Cândido-Júnior, F. A. M. Sales, S. N. Costa, P. De Lima-Neto, D. L. Azevedo, and E. W. S. Caetano, Chem. Phys. Lett. 512, 208 (2011).

${ }^{21}$ A. M. Silva, B. P. Silva, F. A. M. Sales, V. N. Freire, E. Moreira, U. L. Fulco, E. L. Albuquerque, F. F. Maia, and E. W. S. Caetano, Phys. Rev. B 86, 195201 (2012).

${ }^{22}$ S. N. Costa, F. A. M. Sales, V. N. Freire, F. F. Maia, E. W. S. Caetano, L. O. Ladeira, E. L. Albuquerque, and U. L. Fulco, Cryst. Growth Des. 13, 2793 (2013).

${ }^{23}$ J. F. F. Maia, V. N. Freire, E. W. S. Caetano, D. L. Azevedo, F. A. M. Sales, and E. L. Albuquerque, J. Chem. Phys. 134, 175101 (2011).

${ }^{24}$ F. Ortmann, K. Hannewald, and F. Bechstedt, J. Phys. Chem. B 112, 1540 (2008).

${ }^{25}$ R. L. Willett, K. W. Baldwin, K. W. West, and L. N. Pfeiffer, Proc. Natl. Acad. Sci. U.S.A. 102, 7817 (2005).

${ }^{26}$ M. Forster, M. S. Dyer, M. Persson, and R. Raval, J. Am. Chem. Soc. 131, 10173 (2009).

${ }^{27}$ S. Monti, C. Li, and V. Carravetta, J. Phys. Chem. C 117, 5221 (2013).

${ }^{28}$ J. Reichert, A. Schiffrin, W. Auwärter, A. Weber-Bargioni, M. Marschall, M. Dell'Angela, D. Cvetko, G. Bavdek, A. Cossaro, A. Morgante, and J. V. Barth, ACS Nano 4, 1218 (2010).

${ }^{29}$ S. Fischer, A. C. Papageorgiou, M. Marschall, J. Reichert, K. Diller, F. Klappenberger, F. Allegretti, A. Nefedov, C. Wöll, and J. V. Barth, J. Phys. Chem. C 116, 20356 (2012).

${ }^{30}$ M. Smerieri, L. Vattuone, T. Kravchuk, D. Costa, and L. Savio, Langmuir 27, 2393 (2011)

${ }^{31}$ A. Naitabdi and V. Humblot, Appl. Phys. Lett. 97, 223112 (2010).

${ }^{32}$ S. J. Jung, D. H. Kim, S. M. Jeon, S. Hong, and S. Kim, J. Phys. Chem. C 115, 4636 (2011).

${ }^{33}$ M. Oda and T. Nakayama, Jpn. J. Appl. Phys. 45, 8939 (2006).

${ }^{34}$ M. Oda and T. Nakayama, Appl. Surf. Sci. 244, 627 (2005).

${ }^{35}$ M. A. Stroscio and M. Dutta, Proc. IEEE 93, 1772 (2005).

${ }^{36}$ M. Irimia-Vladu, N. S. Sariciftci, and S. Bauer, J. Mater. Chem. 21, 1350 (2011).

${ }^{37}$ V. Parpura, Nat. Nano 7, 143 (2012)

${ }^{38}$ H. Y. Fan, Rep. Prog. Phys. 19, 107 (1956).

${ }^{39}$ A. Fox, Optical Properties of Solids, Oxford Master Series in Condensed Matter Physics (Oxford University Press, 2001).

${ }^{40}$ D. Segall, P. J. D. Lindan, M. J. Probert, C. J. Pickard, P. J. Hasnip, S. J. Clark, and M. C. Payne, J. Phys.: Condens. Matter. 14, 2717 (2002).

${ }^{41}$ S. K. Medeiros, E. L. Albuquerque, F. F. Maia, Jr., E. W. S. Caetano, and V. N. Freire, Chem. Phys. Lett. 435, 59 (2007).

${ }^{42}$ R. Demichelis, P. Raiteri, J. D. Gale, and R. Dovesi, CrystEngComm 14, 44 (2012).

${ }^{43}$ P. Hohenberg and W. Kohn, Phys. Rev. 136, B864 (1964).

${ }^{44}$ W. Kohn and L. J. Sham, Phys. Rev. 140, A1133 (1965).

${ }^{45}$ D. M. Ceperley and B. J. Alder, Phys. Rev. Lett. 45, 566 (1980).

${ }^{46}$ J. P. Perdew and A. Zunger, Phys. Rev. B 23, 5048 (1981).

${ }^{47}$ J. P. Perdew, K. Burke, and M. Ernzerhof, Phys. Rev. Lett. 77, 3865 (1996).

${ }^{48}$ A. Tkatchenko and M. Scheffler, Phys. Rev. Lett. 102, 073005 (2009).

${ }^{49}$ S. Grimme, J. Comput. Chem. 27, 1787 (2006).

${ }^{50}$ J. P. Perdew and Y. Wang, Phys. Rev. B 45, 13244 (1992).

${ }^{51}$ J. S. Lin, A. Qteish, M. C. Payne, and V. Heine, Phys. Rev. B 47, 4174 (1993).

${ }^{52}$ H. J. Monkhorst and J. D. Pack, Phys. Rev. B 13, 5188 (1976).

${ }^{53}$ B. G. Pfrommer, M. Côté, S. G. Louie, and M. L. Cohen, J. Comput. Phys. 131, 233 (1997).

${ }^{54}$ C. S. Wang and W. E. Pickett, Phys. Rev. Lett. 51, 597 (1983).

${ }^{55}$ M. K. Y. Chan and G. Ceder, Phys. Rev. Lett. 105, 196403 (2010).

${ }^{56}$ F. Ortmann, W. G. Schmidt, and F. Bechstedt, Phys. Rev. Lett. 95, 186101 (2005).

${ }^{57}$ F. Ortmann, F. Bechstedt, and W. G. Schmidt, Phys. Rev. B 73, 205101 (2006).

${ }^{58}$ See supplementary material at http://dx.doi.org/10.1063/1.4869179 for tables depicting the atomic fractional coordinates, bond lengths, torsion angles, and hydrogen bond lengths for L-asparagine crystals in both the LDA and GGA+TS approaches, as well as figures displaying the partial densities of states calculated using the GGA+TS exchange-correlation functional. 
${ }^{59}$ A. Seidl, A. Görling, P. Vogl, J. A. Majewski, and M. Levy, Phys. Rev. B 53, 3764 (1996).

${ }^{60}$ J. P. Perdew and M. Levy, Phys. Rev. Lett. 51, 1884 (1983).

${ }^{61}$ L. J. Sham and M. Schlüter, Phys. Rev. B 32, 3883 (1985).

${ }^{62}$ L. J. Sham and M. Schlüter, Phys. Rev. Lett. 51, 1888 (1983).

${ }^{63}$ A. J. Cohen, P. Mori-Sánchez, and W. Yang, Phys. Rev. B 77, 115123 (2008).

${ }^{64}$ X. Zheng, A. J. Cohen, P. Mori-Sánchez, X. Hu, and W. Yang, Phys. Rev. Lett. 107, 026403 (2011).

${ }^{65} \mathrm{~N}$. Vijayan, S. Rajasekaran, G. Bhagavannarayana, R. Ramesh Babu, R. Gopalakrishnan, M. Palanichamy, and P. Ramasamy, Cryst. Growth Des. 6, 2441 (2006).

${ }^{66}$ S. Adhikari and T. Kar, Mater. Chem. Phys. 133, 1055 (2012).

${ }^{67}$ S. Moitra and T. Kar, Cryst. Res. Technol. 45, 70 (2010).

${ }^{68}$ F. Hirshfeld, Theor. Chim. Acta 44, 129 (1977).

${ }^{69}$ R. Kinkar Roy, K. Hirao, S. Krishnamurty, and S. Pal, J. Chem. Phys. 115, 2901 (2001).

${ }^{70}$ R. K. Roy, S. Pal, and K. Hirao, J. Chem. Phys. 110, 8236 (1999).

${ }^{71}$ R. G. Parr and W. Yang, J. Am. Chem. Soc. 106, 4049 (1984).

${ }^{72}$ R. S. Mulliken, J. Chem. Phys. 23, 1833 (1955).

${ }^{73}$ J. P. Foster and F. Weinhold, J. Am. Chem. Soc. 102, 7211 (1980).

${ }^{74}$ R. Bonaccorsi, E. Scrocco, and J. Tomasi, J. Chem. Phys. 52, 5270 (1970).
${ }^{75}$ P. W. Ayers, R. C. Morrison, and R. K. Roy, J. Chem. Phys. 116, 8731 (2002).

${ }^{76}$ R. F. Nalewajski and R. G. Parr, Proc. Natl. Acad. Sci. U.S.A. 97, 8879 (2000).

${ }^{77}$ E. Davidson and S. Chakravorty, Theor. Chim. Acta 83, 319 (1992).

${ }^{78}$ P. Bultinck, C. Van Alsenoy, P. W. Ayers, and R. Carbó-Dorca, J. Chem. Phys. 126, 144111 (2007).

${ }^{79}$ P. Ayers, Theor. Chem. Acc. 115, 370 (2006).

${ }^{80}$ R. G. Parr, P. W. Ayers, and R. F. Nalewajski, J. Phys. Chem. A 109, 3957 (2005).

${ }^{81}$ T. Verstraelen, S. V. Sukhomlinov, V. Van Speybroeck, M. Waroquier, and K. S. Smirnov, J. Phys. Chem. C 116, 490 (2012).

${ }^{82}$ P. Bultinck, S. Fias, C. Van Alsenoy, P. W. Ayers, and R. Carbó-Dorca, J. Chem. Phys. 127, 034102 (2007).

${ }^{83}$ W. C. Guest, N. R. Cashman, and S. S. Plotkin, Phys. Chem. Chem. Phys. 13, 6286 (2011).

${ }^{84}$ G. N. Patargias, S. A. Harris, and J. H. Harding, J. Chem. Phys. 132, 235103 (2010).

${ }^{85}$ A. V. Morozov, T. Kortemme, and D. Baker, J. Phys. Chem. B 107, 2075 (2003).

${ }^{86}$ X. Song, J. Chem. Phys. 116, 9359 (2002). 